Pure and Applied Mathematics Quarterly

Volume 8, Number 1

(Special Issue: In honor of

F. Thomas Farrell and Lowell E. Jones, Part 1 of 2)

$15-51,2012$

\title{
Rigidity and Relative Hyperbolicity of Real Hyperbolic Hyperplane Complements
}

\author{
Igor Belegradek \\ Dedicated to Thomas Farrell and Lowell Jones
}

\begin{abstract}
For $n>3$ we study spaces obtained from finite volume complete real hyperbolic $n$-manifolds by removing a compact totally geodesic submanifold of codimension two. We prove that their fundamental groups are relative hyperbolic, co-Hopf, biautomatic, residually hyperbolic, not Kähler, not isomorphic to lattices in virtually connected real Lie groups, have no nontrivial subgroups with property $(\mathrm{T})$, have finite outer automorphism groups, satisfy Mostow-type Rigidity, have finite asymptotic dimension and rapid decay property, and satisfy Baum-Connes conjecture. We also characterize those lattices in real Lie groups that are isomorphic to relatively hyperbolic groups.
\end{abstract}

Keywords: relatively hyperbolic, hyperplane arrangements, hyperbolic geometry, Mostow rigidity.

\section{INTRODUCTION}

Let $M$ be a (connected) complete finite volume locally symmetric Riemannian manifold of negative sectional curvature, and let $S$ be a (possibly disconnected) compact totally geodesic submanifold of $M$ of codimension two. As noted in Lemma B.1, the manifold $M \backslash S$ is aspherical, and Theorems 1.1-1.2 indicate

Received November 15, 2007.

2000 Mathematics Subject classification. Primary 20F65. Secondary 57R19, 22E40.

This work was partially supported by the NSF grant \# DMS-0503864. 
that the group $\pi_{1}(M \backslash S)$ shares various rigidity properties with lattices in rank one semisimple Lie groups. There are good reasons to focus on codimension two: if $S$ had codimension one, then $M \backslash S$ would be a familiar object, namely, the interior of a compact real hyperbolic manifold with totally geodesic boundary obtained from $M$ by cutting open along $S$, while if $S$ had codimension $>2$, then $M \backslash S$ would no longer be aspherical, and the groups $\pi_{1}(M \backslash S)$ and $\pi_{1}(M)$ would be isomorphic.

It is known that the pair $(M, S)$ is modelled on $\left(\mathbf{C H}^{2}, \mathbf{H}^{2}\right),\left(\mathbf{H}^{n}, \mathbf{H}^{n-2}\right)$, or $\left(\mathbf{C H}^{n}, \mathbf{C H}^{n-1}\right)$, where $\mathbf{C} \mathbf{H}^{n}, \mathbf{H}^{n}$ denotes the hyperbolic $n$-space over $\mathbb{C}$, $\mathbb{R}$, respectively. The purpose of this paper is to study various topological and group-theoretic properties of $M \backslash S$ in the simplest case when $(M, S)$ is modelled on $\left(\mathbf{H}^{n}, \mathbf{H}^{n-2}\right)$. Most results proved here extend to the case $\left(\mathbf{C H}^{n}, \mathbf{C H}^{n-1}\right)$, yet some of the proofs become much harder, and this case shall be treated in [Bel]. While I believe that the arguments in the case $\left(\mathbf{C H}^{n}, \mathbf{C} \mathbf{H}^{n-1}\right)$ can be modified to hold in the "exceptional" case $\left(\mathbf{C H}^{2}, \mathbf{H}^{2}\right)$, this remains to be checked.

Clearly $M \backslash S$ can be identified with the interior of a compact smooth manifold $N$ that is obtained from $M$ by removing a tubular neighborhood of $S$ and chopping off all cusps (in case $M$ is noncompact). There are two kinds of components of $\partial N$ : compact flat manifolds appearing as cusp cross-sections of $M$ (appearing if $M$ is noncompact), and virtually trivial circle bundles over components of $S$, which are locally isometric to the Riemannian product of $S$ and a round circle. It follows from warped product computations of Heintze and Schroeder [Sch91] that $N$ admits a Riemannian metric of sec $\leq 0$ such that sec $<0$ on the interior of $N$, and the boundary $\partial N$ is totally geodesic (see Remark 3.4). In particular, the group $\pi_{1}(N)$ is $C A T(0)$. Furthermore, by compactness of $N$ the HeintzeSchroeder metric is $A$-regular, so deep work of Farrell-Jones [FJ98] implies that that $\pi_{1}(N)$ satisfies Borel's Conjecture.

Results of this paper are summarized below.

Theorem 1.1. Let $M$ be a complete finite volume real hyperbolic $n$-manifold, and $S$ is a compact totally geodesic submanifold of codimension two. Then

(1) $\pi_{1}(N)$ is non-elementary (strongly) relatively hyperbolic, where the peripheral subgroups are fundamental groups of the components of $\partial N$.

(2) the relatively hyperbolic boundary of $\pi_{1}(N)$ is the $(n-1)$-sphere. 
(3) if $n>2$, then $\pi_{1}(N)$ does not split as an amalgamated product or an $H N N$ extension over subgroups of peripheral subgroups of $\pi_{1}(N)$, or over $\mathbb{Z}$.

(4) if $n>2$, then $\pi_{1}(N)$ is co-Hopf.

(5) for any finite subset $S \subset \pi_{1}(N)$ there is a homomorphism of $\pi_{1}(N)$ onto a non-elementary hyperbolic group that is injective on $S$.

(6) $\pi_{1}(N)$ satisfies Strong Tits Alternative.

(7) $\pi_{1}(N)$ is biautomatic.

(8) No nontrivial subgroup of $\pi_{1}(N)$ has Kazhdan property (T).

(9) $\pi_{1}(N)$ is not a Kähler group.

(10) $\pi_{1}(N)$ has finite asymptotic dimension.

(11) $\pi_{1}(N)$ has rapid decay property.

(12) $\pi_{1}(N)$ satisfies Baum-Connes conjecture.

(13) if $n>3$, then $\pi_{1}(N)$ is not isomorphic to the fundamental group of a complete negatively pinched Riemannian manifold.

(14) if $\pi_{1}(N)$ is isomorphic to a lattice $\Lambda$ in a real Lie group $G$, then the identity component $G_{0}$ of $G$ is compact, $\Lambda \cap G_{0}$ is trivial, and $\Lambda$ projects isomorphically onto a finite index subgroup of $G / G_{0}$.

The following is a version of Mostow rigidity.

Theorem 1.2. For $n>2$ and $i=1,2$, suppose that $M_{i}$ is a complete finite volume real hyperbolic $n$-manifold, and $S_{i}$ is a compact totally geodesic submanifold of codimension two. Then any homotopy equivalence $f: M_{1} \backslash S_{1} \rightarrow M_{2} \backslash S_{2}$ induces an isometry $\iota_{f}: M_{1} \rightarrow M_{2}$ taking $S_{1}$ to $S_{2}$ such that the restriction $\iota_{f}: M_{1} \backslash S_{1} \rightarrow M_{2} \backslash S_{2}$ is homotopic to $f$. Moreover, $\iota_{f}$ is uniquely determined by the homotopy class of $f$.

Corollary 1.3. For $n>2$, if $M$ is a complete finite volume real hyperbolic manifold, and $S$ is a compact totally geodesic submanifold of codimension two, then the correspondence $f \rightarrow \iota_{f}$ induces an isomorphism of the outer automorphism group of $\pi_{1}(M \backslash S)$ onto the group of isometries of $M$ that map $S$ to itself. In particular, the outer automorphism group of $\pi_{1}(M \backslash S)$ is finite.

A key technical result of this paper is part (1) of Theorem 1.1. Similarly to Heintze-Schroeder computation, we show that $M \backslash S$ admits a finite volume complete Riemannian metric whose sectional curvature is bounded above by a negative constant. Then (1) and (2) of Theorem 1.1 follow from the closer look 
at properties of the metric; in fact it satisfies Gromov's definition of relative hyperbolicity, as elaborated in [Bow]. It is worth mentioning that previously Fujiwara [Fuj88] constructed a finite volume complete Riemannian metric on $M \backslash S$ with sectional curvatures within $[-1,0)$, but it is unclear to me how to use Fujiwara's metric to prove relative hyperbolicity.

Alternatively, part (1) of Theorem 1.1 can be deduced by combining results of Heintze, Schroeder [Sch91], Kapovich-Leeb [KL95], and Druţu-Osin-Sapir [DS05b]. Details are given in Appendix D, but briefly the universal cover $\tilde{N}$ of $N$ with Heintze-Schroeder's metric is a $C A T(0)$ space that has negative curvature away from $\partial \tilde{N}$, and path components of $\partial \tilde{N}$ are isometric either to $\mathbb{R}^{n-1}$ or $\mathbb{R} \times \mathbf{H}^{n-2}$. Then an argument of Kapovich-Leeb [KL95] shows that $\tilde{N}$ is asymptotically treegraded with respect to $\partial \tilde{N}$, which by results of Druţu-Osin-Sapir [DS05b] implies that $\pi_{1}(N)$ is hyperbolic relative the fundamental groups of components of $\partial N$. It is unclear to me whether this proof also gives part (2) of Theorem 1.1.

Part (14) of Theorem 1.1 follows from the following characterization of lattices in Lie groups that are relatively hyperbolic.

Theorem 1.4. Let $G$ be a real Lie group with identity component $G_{0}$, let $\Lambda$ be a lattice in $G$. Then $\Lambda$ is isomorphic to a non-elementary relatively hyperbolic group if and only if one of the following is true:

(i) $G_{0}$ is compact and $G / G_{0}$ is isomorphic to a non-elementary relatively hyperbolic group;

(ii) $G / G_{0}$ is finite, and $G$ contains a compact normal subgroup $K$ such that $K \subset G_{0}$ and $G_{0} / K$ is a simple noncompact $\mathbb{R}$-rank one Lie group with trivial center.

Remark 1.5. The assertions (i), (ii) of Theorem 1.4 do not mention $\Lambda$, thus relative hyperbolicity of lattices in $G$ can be read off $G$. However, it is instructive to see where $\Lambda$ is "hidden": if $\Lambda_{0}:=\Lambda \cap G_{0}$, then one shows that

- $\Lambda_{0}$ is a lattice in $G_{0}$,

- $G_{0}$ is compact if and only if $\Lambda_{0}$ is finite,

- $\Lambda / \Lambda_{0}$ is a finite index subgroup of $G / G_{0}$,

- the restriction of the projection $G \rightarrow G / K$ to $\Lambda$ has finite kernel. 
None of the results of this paper is truly hard to prove and they draw heavily on various (often deep) works available in the literature. A few harder questions are below.

Let $M$ be a compact locally symmetric irreducible $n$-manifold of sec $\leq 0$ and $S$ is a compact totally geodesic submanifold of codimension two (where we assume $n>3$, else the answers to the questions below are known to be "yes").

Question 1.6. Is $\pi_{1}(M \backslash S)$ quasi-isometricaly rigid?

Theorem 1.2 suggests that the answer might be "yes". Recall that for irreducible nonuniform lattices in semisimple real Lie groups with finite center quasi-isometry implies commensurability (as proved by Schwartz, Eskin et al, see [Far97] for details).

Question 1.7. Is $\pi_{1}(M \backslash S)$ Hopfian? residually finite? linear?

There is currently no general method of establishing Hopf property for relatively hyperbolic groups. Nevertheless, since the peripheral subgroups of $\pi_{1}(M \backslash$ $S)$ are well-understood and quite rigid, the methods of [Sel99, DS08] may suffice to imply that $\pi_{1}(M \backslash S)$ is Hopf.

For $(M, S)$ modelled on $\left(\mathbf{X}_{n}, \mathbf{X}_{n-1}\right)$ where $\mathbf{X}_{n}$ is the symmetric spaces corresponding to $S O(n, 2)$, Toledo [Tol93] used Raghunathan's work [Rag84] to show that if $n \geq 4$ and $n$ is even, then $\pi_{1}(M \backslash S)$ is not residually finite.

If $\pi_{1}(M \backslash S)$ is hyperbolic relative to the fundamental groups of the ends of $M \backslash$ $S$, as happens when $(M, S)$ is modelled on $\left(\mathbf{H}^{n}, \mathbf{H}^{n-2}\right)$ or $\left(\mathbf{C} \mathbf{H}^{n}, \mathbf{C} \mathbf{H}^{n-1}\right)$, then Part (5) of Theorem 1.1, which is based on relatively hyperbolic Dehn Surgery theorem [Osi07] (cf. [GM08]), implies that $\pi_{1}(M \backslash S)$ is residually hyperbolic. In particular, if $\pi_{1}(M \backslash S)$ is not residually finite, then there exists a hyperbolic group that is not residually finite, which illustrates the difficulty of Question 1.7.

Structure of the paper. In Section 2 we write the real hyperbolic metric in cylindrical coordinates and also show that $\partial N$ is a virtually trivial circle bundle over $S$. Section 3 contains a curvature computation, which is then used in Section 4 to prove parts (1)-(2) of Theorem 1.1. Theorems 1.1, 1.2, 1.4 are proved in Sections 5, 6, 7, respectively. Appendix A contains definitions and basic results on relatively hyperbolic groups. A Morse-theoretic lemma describing topology of the universal cover of $M \backslash S$ is proved in Appendix B. In Appendix C we review 
(and also correct) some curvature computation for a multiply-warped product metric that were worked out in [BW04]. Appendix D contains another proof of part (1) of Theorem 1.1.

\section{REAL HYPERBoliC SPACE IN CYLINDRICAL COORDINATES}

We denote the real hyperbolic metric on $\mathbf{H}^{n}$ by $\mathbf{h}_{n}$. Given a totally geodesic subspace $\mathbf{H}^{n-2} \subset \mathbf{H}^{n}$, denote the distance to $\mathbf{H}^{n-2}$ by $r$, and write $\mathbf{h}_{n}$ as $d r^{2}+\rho_{r}$, where $\rho_{r}$ is the induced metric on the $r$-tube $F(r)$ around $\mathbf{H}^{n-2}$. It is well-known that $\rho_{r}$ is the Riemannian product of $\sinh ^{2}(r) d \theta^{2}$ and $\cosh ^{2}(r) \mathbf{h}_{n-2}$ where $d \theta^{2}$ is the round metric on the unit circle, denoted $\mathbf{S}^{\mathbf{1}}$. This fact is crucial for what follows hence we shall outline a proof (which apparently is not recorded in the literature).

The orthogonal projection $\pi: \mathbf{H}^{n} \rightarrow \mathbf{H}^{n-2}$ is a fiber bundle whose fibers are totally geodesic 2-planes. Restricting $\pi$ to $F(r)$ gives a circle bundle $F(r) \rightarrow$ $\mathbf{H}^{n-2}$. This defines a splitting of the tangent bundle $T F(r)=\mathcal{V}(r) \oplus \mathcal{H}(r)$, where $\mathcal{V}(r)$ is tangent to the circle fibers, and $\mathcal{H}(r)$ is the orthogonal complement of $\mathcal{V}(r)$. Fix a point $z \in F(r)$. The metric on $\mathcal{V}(r)$ at $z$ can be computed inside the totally geodesic 2-plane $\pi^{-1}(\pi(z))$, and using the polar coordinates description of the hyperbolic 2-plane $d r^{2}+\sinh ^{2}(r) d \theta^{2}$, we conclude that the induced metric on $\mathcal{V}(r)$ is $\sinh ^{2}(r) d \theta^{2}$. A key feature of the real hyperbolic case is that at each point $z \in F(r)$ the subbundle $\mathcal{H}(r)$ is tangent to the codimension one totally geodesic subspace spanned by $\mathbf{H}^{n-2}$ and $z$. Thus the induced metric on $\mathcal{H}(r)$ at $z$ can be computed inside this codimension one subspace whose metric can be written as $d r^{2}+\cosh ^{2}(r) \mathbf{h}_{n}$, so that the induced metric on $\mathcal{H}(r)$ at $z$ is $\cosh ^{2}(r) \mathbf{h}_{n}$. Therefore, the induced metric in $F(r)$ is a Riemannian submersion metric with base $\cosh (r) \mathbf{H}^{n-2}$ and fiber $\sinh (r)$-multiple of the unit circle. In this metric the circle fibers are closed geodesics, because this can be checked in the totally geodesic 2-plane $\pi^{-1}(\pi(z))$, where it is a tautology that any curve is a geodesic in the induced metric on itself. The $A$-tensor of the Riemannian submersion vanishes because $\mathcal{H}(r)$ is an integrable distribution being tangent to the foliation obtained by intersecting $F(r)$ with the codimension one totally geodesic subspaces containing $\mathbf{H}^{n-2}$. Thus the Riemannian submersion is locally a product, and hence globally a product as $\mathbf{H}^{n-2}$ is simply-connected. In summary, the induced metric on $F(r)$ is the Riemannian product of $\cosh (r) \mathbf{H}^{n-2}$ 
and $\sinh (r)$-multiple of the unit circle. The isometry group of $F(r)$ is the product of the isometry groups of the factors, i.e. $\operatorname{Iso}\left(\mathbf{H}^{n-2}\right) \times O(2)$.

Now if $\Gamma$ a torsion-free discrete isometry group of $\mathbf{H}^{n}$ stabilizing a codimension two totally geodesic subspace $\mathbf{H}^{n-2}$, then $\Gamma$ acts isometrically, freely, and properly discontinuously on $F(r)$. Hence $F(r) / \Gamma$ is a flat Euclidean circle bundle over $\cosh (r)$-multiple of $\mathbf{H}^{n-2} / \Gamma$, where "Euclidean" means that the holonomy of the flat connection lies in $O(2)$.

We shall need the following fact that does not seem to be in the literature.

Proposition 2.1. If $X$ is a connected manifold with finitely generated fundamental group, then any flat Euclidean circle bundle over $X$ becomes trivial, as an $O(2)$-circle bundle, after passing to a finite Galois cover. In particular, the fundamental group of the total space has a finite index normal subgroup isomorphic to $\mathbb{Z} \times \pi_{1}(X)$.

Proof. If $\tilde{X}$ denotes the universal cover of $X$, then any flat Euclidean circle bundle over $X$ is a $\pi_{1}(X)$-quotient of $\tilde{X} \times S^{1}$, where $\pi_{1}(X)$ acts by covering automorphisms on the first factor, and via the holonomy homomorphism on the second factor. Passing to the orientation cover of the bundle cover, we can assume that the bundle is orientable, while the fundamental group of the base is still finitely generated. Orientable flat Euclidean circle bundles are classified by holonomy $\pi_{1}(X) \rightarrow S O(2)$, so let $\varphi$ be the holonomy homomorphism of the flat structure. Since $S O(2)$ is abelian, $\varphi$ factors through $\bar{\varphi}: H_{1}(X) \rightarrow S O(2)$. Write $H_{1}(X)=\mathbb{Z}^{k} \oplus T$ where $T$ is the (finite) torsion subgroup, and deform the $\mathbb{Z}^{k}$ summand to the trivial subgroup inside $S O(2)$ without changing $\bar{\varphi}_{\mid T}$. (To do so choose a generating set $s_{1}, \ldots s_{k}$ for $\mathbb{Z}^{k}$, fix arbitrary paths $p_{i}(t)$ in $S O(2)$ from $\bar{\varphi}\left(s_{i}\right)$ to 1 , and define $\bar{\varphi}_{t}\left(s_{i}\right)=p_{i}(t)$ and $\left.\bar{\varphi}_{t \mid T}=\bar{\varphi}_{\mid T}\right)$. The endpoint is $\bar{\varphi}_{1}: \pi_{1}(X) \rightarrow S O(2)$ whose image $\bar{\varphi}_{1}(T)$ is finite. Precomposing with the abelianization $\pi_{1}(X) \rightarrow H_{1}(X)$ yields a path in the representation variety $\operatorname{Hom}\left(\pi_{1}(X), S O(2)\right)$ from $\varphi$ to a homomorphism with image $\bar{\varphi}(T)$. By the covering homotopy theorem, the corresponding flat $S O(2)$-bundles have isomorphic underlying $S O(2)$-bundles. Clearly, if a bundle corresponds to a homomorphism $\pi_{1}(X) \rightarrow S O(2)$ with finite image, then it becomes trivial in the cover that corresponds to the kernel of the homomorphism. 
Remark 2.2. A quicker but less elementary way to see that the bundle $\partial N \rightarrow S$ is virtually trivial is to note that $\partial N$ is a totally geodesic submanifold in the nonpositively curved Heintze-Schroeder metric on $N$, so that the fundamental group of each component of $\partial N$ is $C A T(0)$, which implies that the centralizers virtually split [BH99, Theorem 1.1(iv), page 439].

\section{Curvature computation}

The curvature computations done in this section are straightforward and they could have been omitted had this paper been written for differential geometers. Since we expect more diverse readership, full details are given. Also instead of using ad hoc arguments, we find it more illuminating to rely on general curvature formulas developed in [BW04] and reviewed in Appendix C. This section could serve as a gentle introduction to delicate curvature computations in [Bel], which use Appendix $\mathrm{C}$ in essential way.

Given positive functions $v, h$, we write the Riemannian product metric of $v(r) \mathbf{S}^{\mathbf{1}}$ and $h(r) \mathbf{H}^{n-2}$ as $\lambda_{r, v, h}=v^{2}(r) d \theta^{2}+h^{2}(r) \mathbf{h}_{n-2}$. Consider the metric $\lambda_{v, h}=d r^{2}+\lambda_{r, v, h}$ on $I \times F$, where $I$ is an open interval, and $F$ is an underlying smooth manifold of $\mathbf{S}^{\mathbf{1}} \times \mathbf{H}^{n-2}$. Thus if $v(r)=\sinh (r), h(r)=\cosh (r)$ and $I \subseteq(0, \infty)$, then $\lambda_{v, h}$ coincides with the real hyperbolic metric $\mathbf{h}_{n}$. For brevity we sometimes suppress $v, h$ and label tensors associated with $\lambda_{v, h}, \lambda_{v, h, r}$ by $\lambda$, $\lambda_{r}$, respectively, and also denote by $K$ the sectional curvature of $\lambda$.

Next we define a local orthonormal frame on $I \times F$ in which the curvature of $\lambda_{v, h}$ will be computed. Denote $\frac{\partial}{\partial r}$ by $\partial_{r}$, and $\frac{\partial}{\partial \theta}$ by $X_{1}$. Fix $z \in I \times F$ and let $w \in \mathbf{H}^{n-2}$ be the image of $z$ under the projection to the last coordinate $p: I \times F \rightarrow \mathbf{H}^{n-2}$. Consider an arbitrary orthonormal frame $\left\{\check{X}_{i}\right\}$, with $1<$ $i<n$, defined on a neighborhood of $w$ in $\mathbf{H}^{n-2}$ such that $\left[\check{X}_{i}, \check{X}_{j}\right]$ vanishes at $w$ for all $i, j$. (By a standard argument any orthonormal basis in $T_{w} \mathbf{H}^{n-2}$ can be extended to some $\left\{\check{X}_{i}\right\}$ as above). Let $X_{i}$ be the vector field on $I \times F$ obtained by lifting $\check{X}_{i}$ via the coordinate inclusions $\mathbf{H}^{n-2} \rightarrow I \times F$. Then $\partial_{r}, X_{1}, \ldots, X_{n-1}$ is an orthogonal frame near $z$ such that

(1) $\left\langle X_{1}, X_{1}\right\rangle_{\lambda}=v^{2}$, and $\left\langle X_{i}, X_{i}\right\rangle_{\lambda}=h^{2}$ for $i>1$.

(2) $\left[X_{i}, X_{j}\right]=0=\left[X_{i}, \partial_{r}\right]$ at $z$ for all $i, j$, 
where all brackets vanish at $z$ as the manifold is smoothly a product. The corresponding orthonormal frame $\partial_{r}, Y_{1}=\frac{1}{v} X_{1}, Y_{i}=\frac{1}{h} X_{i}, i>1$ has the following properties:

(i) $\left[Y_{i}, Y_{j}\right]=\frac{1}{h^{2}}\left[X_{i}, X_{j}\right]=0$ for $i, j>1$,

(ii) $\left[Y_{i}, Y_{1}\right]=\frac{1}{h v}\left[X_{i}, X_{1}\right]=0$,

where the first equalities in (i), (ii) hold because any function of $r$ has zero derivative in the direction of $X_{i}$.

Both $v(r) \mathbf{S}^{\mathbf{1}}$ and $h(r) \mathbf{H}^{n-2}$ have constant sectional curvature so computing the curvature tensor of $R_{\lambda_{r}}$ is straightforward, e.g. it follows from [KN63, Corollary V.2.3] and [Bes87, Theorem 9.28] that up to symmetries of the curvature tensor the only nonzero components of $R_{\lambda_{r}}$ are the sectional curvatures of $Y_{i} Y_{j}$ planes with $1<i<j<n$, which is $-h^{-2}$.

Combining this with the fact that all brackets $\left[Y_{i}, Y_{j}\right]$ vanish, we see from Appendix $\mathrm{C}$ that up to symmetries of the curvature tensor the only nonzero components of the curvature tensor $R_{\lambda}$ are

$$
\begin{gathered}
\sec _{\lambda}\left(Y_{i}, Y_{1}\right)=-\frac{h^{\prime} v^{\prime}}{h v}, \quad \sec _{\lambda}\left(Y_{i}, \partial_{r}\right)=-\frac{h^{\prime \prime}}{h}, \quad \sec _{\lambda}\left(Y_{1}, \partial_{r}\right)=-\frac{v^{\prime \prime}}{v}, \\
\sec _{\lambda}\left(Y_{i}, Y_{j}\right)=-\frac{1}{h^{2}}-\left(\frac{h^{\prime}}{h}\right)^{2} \text { where } 1<i<j<n .
\end{gathered}
$$

Hence the bivectors $Y_{k} \wedge Y_{l}, Y_{i} \wedge \partial_{r}$ with $k<l$ diagonalize the curvature operator, and hence global maxima and minima of $\sec _{\lambda}$ are attained on the coordinate planes $Y_{k} Y_{l}, Y_{i} \partial_{r}$, see e.g. [Pet06, Chapter 3, Section 1]. Thus to achieve $\sec _{\lambda}<0$ we just need to choose $h, v$ increasing and satisfying $h^{\prime \prime}>0, v^{\prime \prime}>0$.

Let $M, S$ be as in Theorem 1.1. We are going to modify the incomplete real hyperbolic metric on the ends of $M \backslash S$ near $S$. Suppose $\epsilon$ is less than the half of the normal injectivity radius of $S$ in $M$, and let $I=(-\infty, \epsilon)$. Let $\rho \gg 1$ be a parameter. We let $h(r)=v(r)=e^{r}$ on $(-\infty,-\rho]$, let $h(r)=\cosh (r)$, $v(r)=\sinh (r)$ on $[\epsilon, \infty)$, and then interpolate on $[-\rho, \epsilon]$ to ensure that $h, v$ are smooth and $h^{\prime}, v^{\prime}, h^{\prime \prime}, v^{\prime \prime}$ are positive on $[-\rho, \epsilon]$, which is clearly possible for sufficiently large $\rho$ (e.g. choose $\rho$ so that the tangent lines to $e^{r}$ at $r=-\rho$ and to $\cosh (r), \sinh (r)$ at $r=\epsilon$ intersect on $(-\rho, \epsilon))$. Consider the corresponding metric $\lambda=\lambda_{v, h}$. By (3.1)-(3.2) one checks that $\sec _{\lambda} \leq-1$ if $r \leq-\rho$, and of course $\sec _{\lambda}=-1$ if $r \geq \epsilon$. So compactness of $[-\rho, \epsilon]$ implies that $\sec _{\lambda}$ is bounded 
above by a negative constant depending only on $\rho, \epsilon$. The metric $\lambda$ descends to a metric on the ends of $M \backslash S$ near $S$; the metric will be also denoted $\lambda=\lambda_{v, h}$.

Remark 3.3. Every end of $M \backslash S$ with metric $\lambda_{v, h}$ admits a Riemannian submersion onto $(-\infty, 0]$, and we refer to its fibers as cross-sections. In turn, every cross-section is the total space of a Riemannian submersion with fiber $v \mathbf{S}^{\mathbf{1}}$ and base $h B$, where $B$ is a component of $S$. The volume of the cross-section can be computed by Fubini's theorem for Riemannian submersions [Sak96, Corollary 5.7], and since $v \mathbf{S}^{\mathbf{1}}$ has volume $2 \pi v$, the volume of the cross-section is $2 \pi v h^{2 n-2} \operatorname{vol}(B)$. By the same result, the volume of the portion of the end corresponding to $[r, 0]$ is $2 \pi \operatorname{vol}(B) \int_{r}^{0} v h^{2 n-2} d r$ and the volume of the end is obtained by taking $r$ to $-\infty$. It follows that the ends have finite volume provided $h$ is bounded and $\int_{-\infty}^{r_{0}} v<\infty$, which holds for $\lambda_{v, h}$. Thus if the ends of $M \backslash S$ that approach $S$ are given the metric $\lambda_{v, h}$, then $M \backslash S$ has finite volume.

Remark 3.4. (1) By choosing $v, h$ equal to small constants on $(-\infty, \rho]$ one recovers the Heintze-Schroeder's metric [Sch91] of sec $\leq 0$ that is a product metric at the ends that approach $S$.

(2) By making $v(r)=e^{r}$ and $h(r)=e^{r}+\tau$ on $(-\infty, \rho]$, for some small positive $\tau=\tau(\rho, \epsilon)$ one recovers Fujiwara's result [Fuj88] that $M \backslash S$ admits a complete finite volume metric of sectional curvature within $[-1,0)$.

Remark 3.5. If $n \leq 3$, then $\sec _{\lambda}$ is bounded between two negative constants because $Y_{i} Y_{j}$ with $1<i<j<n$ is the only coordinate plane on which there is no lower curvature bound, and $1<i<j<n$ cannot occur when $n \leq 3$.

\section{Proving Relative hyperbolicity}

We refer to Appendix A for basic information on relatively hyperbolic groups. As is briefly explained in [Bow, Section 6], Gromov's definition of relative hyperbolicity (stated somewhat informally in [Gro87, Definition 8.6.A]) is equivalent to Definition A.1, which is adopted in the present paper. Proposition 4.1 below is essentially the assertion that Gromov's definition implies Definition A.1 stated for group actions on negatively curved Hadamard manifolds. Unfortunately, we could not find the precise statement we need in the literature, so we supply a proof. 
Proposition 4.1. Let $G$ be a group acting properly discontinuously and isometrically on a complete simply-connected Riemannian $n$-manifold $X$ with $\sec (X) \leq$ -1 and $n \geq 2$. Suppose that $X$ contains a $G$-invariant family of horoballs $\mathcal{B}$ such that

(1) for some $r>0$ the distance between any two distinct horoballs in $\mathcal{B}$ is $\geq r$,

(2) $G$ acts cocompactly on the space $X_{0}$ obtained by removing from $X$ the interiors of the horoballs in $\mathcal{B}$.

Then $G$ is a non-elementary relatively hyperbolic, relative to the set of stabilizers of the horoballs in $\mathcal{B}$. The relatively hyperbolic boundary of $G$ is $\partial X$, which is homeomorphic to $(n-1)$-sphere.

Proof. Let $\Pi$ be the set of centers of horoballs in $\mathcal{B}$. First, we show that any point of $\Pi$ is bounded parabolic. In fact, if $z$ is the center of a horoball $B \in \mathcal{B}$, then the stabilizer $\operatorname{Stab}_{G}(B)$ of $B$ in $G$ is a parabolic subgroup fixing $z$ and acting cocompactly on the horosphere $\partial B$. (Indeed, $\operatorname{Stab}_{G}(B)$ fixes $z$, and hence $\operatorname{Stab}_{G}(B)$ is an elementary subgroup that acts cocompactly and properly discontinuously on the horosphere $\partial B$, which is noncompact. In particular, $\operatorname{Stab}_{G}(B)$ is infinite. Hence either $\operatorname{Stab}_{G}(B)$ is a parabolic subgroup, or else $\operatorname{Stab}_{G}(B)$ stabilizes a geodesic in $X$ emanating from $z$; the latter alternative is impossible because then $\operatorname{Stab}_{G}(B)$ would have to fix the point of $X$ where the geodesic intersects $\partial B$.)

Second, any point of $y \in \partial X \backslash \Pi$ is conical limit point. Indeed, any ray emanating from $y$ intersects $X_{0}$ in a unbounded set (else the ray would eventually stay in one horoball, so $y$ would be its center contradicting $y \notin \Pi)$. Take $x_{i} \in X_{0}$ that lie on the ray and tend to $y$. Since $X_{0} / G$ is compact, it has finite diameter $D$, so there is an infinite $G$-orbit $g_{i}(x)$ with $\operatorname{dist}\left(x_{i}, g_{i}(x)\right) \leq D$, hence $y$ is conical.

By Definition A.1 it follows that $G$ is hyperbolic relative to its maximal parabolic subgroups, and the relatively hyperbolic boundary equals to $\partial X$, which is an $(n-1)$-sphere, in particular, $\partial X$ contains more than two points, so $G$ is non-elementary.

Next observe that maximal parabolic subgroups of $G$ are precisely the subgroups $\operatorname{Stab}_{G}(z), z \in \Pi$. Indeed, no conical limit point can be fixed by a parabolic subgroup [Bow99, Proposition 3.2], so if $z$ is the fixed point of a maximal parabolic subgroup, then $z \in \Pi$ and the maximal parabolic subgroup lies 
in $\operatorname{Stab}_{G}(z)$. Conversely, any point $z \in \Pi$ is fixed by the parabolic subgroup $\operatorname{Stab}_{G}(z)$, which is contained in a maximal parabolic subgroup whose unique fixed point must be $z$.

Finally, note that if the horoball $B \in \mathcal{B}$ is centered at $z$, then $\operatorname{Stab}_{G}(B)$ equals to the stabilizer $\operatorname{Stab}_{G}(z)$ of $z$ in $G$ : the inclusion $\operatorname{Stab}_{G}(B) \subseteq \operatorname{Stab}_{G}(z)$ is obvious, and if $g(z)=z$, then $g(B)$ and $B$ are concentric horoballs in $\mathcal{B}$, hence $g(B)=B$ by the assumption (1).

A neighborhood $E$ of an end in a complete Riemannian manifold is called a cusp neighborhood if $E$ admits a Riemannian submersion onto [0, $\infty)$, and there exists a constant $K$ such that the "holonomy" diffeomorphism $h_{t}$ from the fiber over $\{0\}$ to the fiber over $\{t\}$ is $K$-Lipschitz for each $t$.

To make sense of the above definition recall that Riemannian submersions of complete manifolds are smooth fiber bundles, so fibers of $E \rightarrow[0, \infty)$ are closed smooth submanifolds, and we denote the fiber over $\{t\}$ by $F_{t}$. Through every point of $E$ there exists a unique horizontal geodesics ray that start at $F_{0}$; the ray intersects each $F_{t}$ orthogonally and projects isometrically onto $[0, \infty)$. Pushing along horizontal geodesics ray defines the diffeomorphism $h_{t}: F_{0} \rightarrow F_{t}$.

Theorem 4.2. For $n \geq 2, k \geq 1$, suppose that $V$ is a complete Riemannian $n$-manifold with $k$ ends, which have disjoint cusp neighborhood $E_{1}, \ldots, E_{k}$. Let $q: \tilde{V} \rightarrow V$ denote the universal cover of $V$. Then

(1) If $\sec (V) \leq 0$, then for each $i$, the inclusion $E_{i} \rightarrow V$ is $\pi_{1}$-injective, and every component of $q^{-1}\left(E_{i}\right)$ is a horoball.

(2) If $\sec (V)$ is bounded above by a negative constant, and each of the submersions $E_{i} \rightarrow[0, \infty)$ has compact fibers, then $\pi_{1}(V)$ is non-elementary relatively hyperbolic, relative to the conjugates of $\pi_{1}\left(E_{1}\right), \ldots \pi_{1}\left(E_{k}\right)$ in $\pi_{1}(V)$, and the relatively hyperbolic boundary is the ideal boundary of $\tilde{V}$, which is homeomorphic to $S^{n-1}$.

Proof. Fix an end of $V$, and let $E \in\left\{E_{1}, \ldots, E_{k}\right\}$ be the corresponding cusp neighborhood of the end. Fix an arbitrary component $C$ of $q^{-1}(E)$, so that $q$ restricts to a covering $C \rightarrow E$. We next show that $C$ is a horoball. The Riemannian submersion $E \rightarrow[0, \infty)$ lifts to a Riemannian submersion $C \rightarrow[0, \infty)$, whose fibers are denoted $\tilde{F}_{t}$. The diffeomorphisms $h_{t}$ lifts to a diffeomorphism $\tilde{h}_{t}: \tilde{F}_{0} \rightarrow \tilde{F}$ defined by pushing along horizontal geodesic rays of $C \rightarrow[0, \infty)$. 
Since each $\tilde{h}_{t}$ is clearly $K$-Lipschitz, any two horizontal geodesic rays of the submersion $C \rightarrow[0, \infty)$ are asymptotic in $\tilde{V}$ (because the Hausdorff distance between the rays is bounded above by $K$ times the distance between their initial points in $\tilde{F}_{0}$ ). So the horizontal geodesic rays define a point at infinity of $\tilde{V}$. The horospheres in $\tilde{V}$ centered at the point are orthogonal to the horizontal rays in $C$ and hence by dimension reasons their tangent spaces coincide with tangent spaces of fibers of $C \rightarrow[0, \infty)$. Since a foliation is determined by its tangent subbundle, the foliations of $C$ by horospheres and by the fibers $F_{t}$ coincide. As fibers and horospheres are codimension one closed submanifolds of $\tilde{V}$, every fiber is a closed and open subset of a horosphere, so each $\tilde{F}_{t}$ equals to a horosphere. Thus $C$ is the union of the horospheres that intersect a fixed horizontal geodesic ray in $C$, i.e. $C$ is a horoball. Since horoballs are contractible, the inclusion $E \rightarrow V$ is $\pi_{1}$-injective, which proves (1).

Let $G:=\pi_{1}(V)$ and $X:=\tilde{V}$, and let $\mathcal{B}$ be the set of horoballs in $X$ that are components of $q^{-1}\left(\cup_{i} E_{i}\right)$. To prove (2) we are going to check that the $G$-action on $X$ satisfies the assumptions of Proposition 4.1. Let $L:=V \backslash \cup_{i} E_{i}$, i.e. $L$ is the $G$-quotient of the space obtained from $X$ by removing all horoballs of $\mathcal{B}$. By assumptions the closure of $L$ in $V$ is a compact manifold with boundary, and distinct horoballs are $r$-separated e.g. by setting $3 r$ equal to the normal injectivity radius of $\partial L$. By Proposition $4.1 G$ is a non-elementary relatively hyperbolic, relative to the set of stabilizers of the horoballs in $\mathcal{B}$. Finally, the stabilizers of horoballs in $\mathcal{B}$ are precisely the conjugates of the subgroups $\pi_{1}\left(E_{1}\right), \ldots, \pi_{1}\left(E_{k}\right)$, which completes the proof.

Proof of (1)-(2) in Theorem 1.1. By Section 3, we equip $M \backslash S$ with a complete metric of sectional curvature bounded above by a negative constant. Each end of $M \backslash S$ corresponding to a cusp of $M$ has a neighborhood with warped product metric $d r^{2}+e^{2 r} d f^{2}$ where $d f^{2}$ is a flat metric on a cusp cross-section. Each end of $M \backslash S$ that approaches $S$ has a neighborhood with metric $d r^{2}+v^{2} d \theta^{2}+$ $h^{2} \mathbf{h}_{n-2}$. In either case the $r$-coordinate projection is a Riemannian submersion with compact fibers, and $h_{t}$ is 1-Lipschitz as $e^{r}, v, h$ are increasing, so these are cusp neighborhoods, and Theorem 4.2 applies.

Remark 4.3. The conclusion that the relatively hyperbolic boundary is a sphere is of interest in itself. Previously known relatively hyperbolic groups with sphere boundaries are the fundamental groups of complete Riemannian orbifolds of finite 
volume and pinched negative curvature, or closed aspherical piecewise hyperbolic locally $C A T(-1)$ manifolds whose links are topological spheres [DJ91]; these manifolds can be produced via the strict hyperbolization of polyhedra [CD95].

\section{Proof of Theorem 1.1}

The parts (1)-(2) are proved in Section 4.

(3) immediately follows from (1) and a result of the author [Bel07, Theorem 1.3], where it was proved that if $N$ is a compact aspherical manifold with incompressible boundary and $\pi_{1}(N)$ is hyperbolic relative to fundamental groups of components of $\partial N$ and $\operatorname{dim}(N)>2$, then $\pi_{1}(N)$ does not split over elementary subgroup of the relatively hyperbolic group $\pi_{1}(N)$.

(4) A group is called co-Hopf if any injective endomorphism is surjective. The co-Hopf property for $\pi_{1}(N)$ follows from (1) and (3) together with a result of Druţu-Sapir [DS08] (see [Bel07, Theorem 1.3] for details). Alternatively, by [Bel07, Theorem 1.5], the co-Hopf property for $\pi_{1}(N)$ follows from (1) together with results of Mineyev-Yaman [MY] who showed that hyperbolicity of $\pi_{1}(N)$ relative to the boundary implies positivity of the relative simplicial volume $\|N, \partial N\|$.

(5) Given a class of groups $\mathcal{C}$, a group $\Gamma$ is called fully residually $\mathcal{C}$ if any finite subset of $\Gamma$ can be mapped injectively by a homomorphism of $\Gamma$ onto a group in $\mathcal{C}$. Osin [Osi07] proved that if all peripheral subgroups of a non-elementary relatively hyperbolic group $G$ are fully residually hyperbolic, then $G$ is fully residually nonelementary hyperbolic. (This result can also be deduced from [GM08] provided $G$ is torsion-free and finitely generated, which is true for $\left.G=\pi_{1}(N)\right)$. Now (5) follows from (1) together with the fact that the peripheral subgroups of $\pi_{1}(N)$ are fully residually finite, and hence fully residually hyperbolic because finite groups are hyperbolic.

To see that peripheral subgroups of $\pi_{1}(N)$ are fully residually finite recall that the components of $\partial N$ corresponding to the cusps of $M$ have finitely generated virtually abelian fundamental groups, and of course, finitely generated abelian groups are residually finite. By Proposition 2.1 the fundamental group of any other component of $\partial N$ contains a finite index subgroup isomorphic to $\mathbb{Z} \times \pi_{1}(B)$ where $B$ is a component of $S$. The groups $\mathbb{Z}, \pi_{1}(B)$ are linear and finitely 
generated, hence so is their product, which implies residual finiteness of $\mathbb{Z} \times \pi_{1}(B)$ by a classical result of Mal'tsev. Thus all components of $\partial N$ have residually finite fundamental groups. Finally, if the class $\mathcal{C}$ closed under finite direct products, then it is immediate that any residually $\mathcal{C}$ group is fully residually $\mathcal{C}$, and we conclude that $\pi_{1}(N)$ is fully residually finite.

(6) A group satisfies the Strong Tits Alternative if any subgroup either contains a nonabelian free group or is virtually abelian. Tukia [Tuk94] (cf. [Gro87, Secton 8.2.F]) proved the following Tits Alternative for relatively hyperbolic groups: a subgroup that does not contain a non-abelian free subgroup is either finite, or virtually- $\mathbb{Z}$, or lies in a peripheral subgroup. Thus it suffices to prove the strong Tits alternative for the peripheral subgroups, which are extensions of nonelementary hyperbolic groups by $\mathbb{Z}$ (the fundamental group of a circle bundle over a closed hyperbolic manifold is such by the homotopy exact sequence of the bundle). Suppose $Q$ is hyperbolic and $p: G \rightarrow Q$ is a quotient with infinite cyclic kernel. If $H \leq G$ does not contain $\mathbb{Z} * \mathbb{Z}$, then neither does $p(H)$, so $p(H)$ is trivial or virtually- $\mathbb{Z}$, which immediately implies that $H$ is virtually abelian of rank $\leq 2$.

(7) According to Rebecchi [Reb01] a relatively hyperbolic group is biautomatic provided its peripheral subgroups are biautomatic. Virtually abelian groups are biautomatic $\left[\mathrm{ECH}^{+} 92\right]$, and so are virtually central extensions of hyperbolic groups [NR97], so (1) implies that $G$ is biautomatic.

(8) Lemma B.1, proved in Appendix B, implies that the kernel $K$ of the surjection $\pi_{1}(M \backslash S) \rightarrow \pi_{1}(M)$ is a (countably generated) free group. If $H$ is a subgroup of $\pi_{1}(M \backslash S)$ with Kazhdan property (T), then it lies in the kernel because any action of $K$ on a real hyperbolic space has a fixed point [dlHV89], while $\pi_{1}(M)$ acts freely. Thus the group $H$ is free, and hence trivial, as nontrivial free groups do not have property $(\mathrm{T})$.

Remark 5.1. Similarly, one could show that $\pi_{1}(N)$ contains no nontrivial simple subgroup for if $K$ is a simple subgroup of $\pi_{1}(N)$, then either $H \subset K$ or $H \cap K=$ $\{1\}$. In the former case $H$ is free hence trivial, as nontrivial free groups are not simple. In the latter case $H$ embeds into $\pi_{1}(M)$, which is residually finite, so that $H$ is residually finite and hence not simple. 
(9) Arguing by contradiction, suppose that $K$ is a compact Kähler manifold with $\pi_{1}(K)$ isomorphic to $\pi_{1}(M \backslash S)$. Since $S$ has codimension two, the inclusion $M \backslash S$ induces a surjection $\pi_{1}(M \backslash S) \rightarrow \pi_{1}(M)$. Precomposing with the isomorphism $\pi_{1}(K) \cong \pi_{1}(M \backslash S)$ we get a surjection $\pi_{1}(K) \rightarrow \pi_{1}(M)$, which is homotopic to a non-constant harmonic map $K \rightarrow M$ [Lab91]. It was independently proved in [CT89, Theorem 7.1] and [JY91, Theorem 3] that the harmonic map $K \rightarrow M$ factors through a closed geodesic or a compact Riemann surface $\Sigma$. Since $\pi_{1}(M)$ is not virtually abelian, the map cannot factor through a geodesic, and $\Sigma$ must have negative Euler characteristic.

Fix a component $C$ of $S$, and let $B$ be the corresponding component of $\partial N$. By Proposition $2.1 B$ is a virtually trivial circle bundle over $C$. So there exists a finite cover $\bar{C} \rightarrow C$ and a $\pi_{1}$-injective map $\bar{C} \rightarrow B$ such that $\bar{C} \rightarrow B$ followed by the bundle projection $B \rightarrow C$ is homotopic to $\bar{C} \rightarrow C$. The inclusion $B \rightarrow N \subset M \backslash S$ is $\pi_{1}$-injective, and furthermore, the inclusion $B \rightarrow \partial N \rightarrow M$ is homotopic to the bundle projection $B \rightarrow C$ followed by the inclusion $C \rightarrow M$. Thus the covering $\bar{C} \rightarrow C \subset M$ homotopy factors through the inclusion $B \rightarrow M$. It follows that $\pi_{1}(K)$ contains a subgroup isomorphic to $\pi_{1}(\bar{C})$, and the above harmonic map maps this subgroup isomorphically onto a finite index subgroup of $\pi_{1}(C) \leq \pi_{1}(M)$. Since the harmonic map factors through $\Sigma$, the group $\pi_{1}(\bar{C})$ injects into $\pi_{1}(\Sigma)$. So $\pi_{1}(\bar{C})$ has cohomological dimension $\leq 2$. Since $\bar{C}$ is aspherical, $\bar{C}$ has dimension $\leq 2$, and hence $\operatorname{dim}(S) \leq 2$.

Nontrivial free groups are not Kähler $\left[\mathrm{ABC}^{+} 96\right.$, Example 1.19] so $\operatorname{dim}(S)>0$. If $S$ is 2 -dimensional, then the above $\pi_{1}$-injective map $\bar{C} \rightarrow \Sigma$ is homotopic to a finite cover, so its image of $\pi_{1}(\Sigma) \rightarrow \pi_{1}(M)$ is commensurable to $\pi_{1}(C) \leq \pi_{1}(M)$, and in particular, $\pi_{1}(\Sigma) \rightarrow \pi_{1}(M)$ is not onto, which is not the case. If $S$ is one dimensional, then $M \backslash S$ is a real hyperbolic 3-manifold with finitely many closed geodesics removed. Then it is a standard observation that $M \backslash S$ can be given negatively pinched metric e.g. by Remark 3.5, hence $N$ is irreducible and atoroidal, so by Thurston's hyperbolization theorem $M \backslash S$ admits a finite volume real hyperbolic metric. If the group $\pi_{1}(M \backslash S)$ is Kähler, then we can repeat the above argument for a harmonic map from $K$ to this real hyperbolic 3 -manifold, that induces a $\pi_{1}$-isomorphism. We then conclude that the group $\pi_{1}(K) \cong \pi_{1}(M \backslash S)$ injects into $\pi_{1}(\Sigma)$. But $\pi_{1}(M \backslash S)$ contains $\mathbb{Z} \oplus \mathbb{Z}$ as a cusp subgroup, which contradicts the fact that $\Sigma$ is has negative Euler characteristic. 
(10) Osin proved in [Osi05] that a relatively hyperbolic group has finite asymptotic dimension, provided all peripheral subgroups have finite asymptotic dimension. It is known (see e.g. [BD08, Corollary 60]) that asymptotic dimension of finitely generated groups is invariant under commensurability. Furthermore, the asymptotic dimension of an extension of finitely generated groups is bounded above by the sum of the asymptotic dimensions of the kernel and the quotient [BD06, Theorem 7], Since $\mathbb{Z}$ has asymptotic dimension 1 , any polycyclic finitely generated group has finite asymptotic dimension, and in particular, this applies to finitely generated nilpotent groups. It remains to deal with extensions whose kernel is $\mathbb{Z}$, and the quotient is word-hyperbolic. For word-hyperbolic groups finiteness of asymptotic dimension is due to Gromov (see [Roe05]).

(11) Drutu-Sapir [DS05a] proved that a relatively hyperbolic group has rapid decay property provided so do all peripheral subgroups. Rapid decay property for groups of polynomial growth (and in particular virtually abelian groups) was established by Jolissaint [Jol90, Proposition 2.1.9] who also proved that the rapid decay property is invariant under commensurability [Jol90, Section 2.1]. So it remains to prove rapid decay property for central extensions with kernel $\mathbb{Z}$ and hyperbolic quotient which was done by Noskov [Nos92].

(12) Lafforgue [Laf02, Corollary 0.0.4] proved Baum-Connes conjecture for groups with rapid decay property that are isomorphic to the fundamental groups of $A$-regular nonpositively curved manifolds. A Riemannian metric is called $A$ regular if for every $k \geq 0$, each component of the $k$ th covariant derivative of the curvature tensor is a bounded function on the manifold. By obvious compactness considerations, any metric that is a product near infinity is $A$-regular, hence the Heintze-Schroeder's metric [Sch91] on $M \backslash S$ is $A$-regular, so the result follows from (11).

Remark 5.2. Farrell-Jones showed that the fundamental group of a complete $A$-regular manifold of sec $\leq 0$ satisfies the Borel's Conjecture [FJ98, Proposition $0.10]$, which says that the $L$ and $K$-theory assembly maps are isomorphisms. In particular, if $n \geq 5$, then any homotopy equivalence of compact manifolds $L \rightarrow N$ that restricts to a homeomorphism $\partial L \rightarrow \partial N$ is homotopic rel boundary to a homeomorphism [FJ98, Addendum 0.5]. 
(13) In a discrete isometry group of a negatively pinched Hadamard manifold the centralizer of any infinite order element is virtually nilpotent. Indeed, an infinite order element is either hyperbolic or parabolic. if the element is hyperbolic, then it has exactly two fixed points at infinity which then must be stabilized by the centralizer. Hence an index two subgroup of the centralizer fixes the two points, and hence acts properly discontinuously on the geodesic joining the points. This shows that the centralizer is virtually cyclic. If the infinite order element is parabolic, then it fixes exactly one point at infinity, and this point then must be fixed by the centralizer. According to Bowditch [Bow93] the centralizer is finitely generated and hence Margulis's Lemma shows it is also virtually nilpotent. If $n>3$, then $\pi_{1}(N)$ has a peripheral subgroup that is an extension with infinite cyclic kernel and non-elementary hyperbolic quotient. Since $\operatorname{Aut}(\mathbb{Z})$ has order two, its index two subgroup centralizes the infinite cyclic kernel, yet the group is not virtually nilpotent, because it surjects onto a non-elementary hyperbolic group.

(14) Suppose that $\pi_{1}(N)$ embeds as a lattice $\Lambda$ into a real Lie group $G$ with identity component $G_{0}$, so Theorem 1.4 applies. Case (ii) is impossible, indeed $\Lambda_{0}:=\Lambda \cap G_{0}$ is isomorphic to a finite index subgroup of $\pi_{1}(N)$, so $\Lambda_{0}$ is torsionfree, hence it projects isomorphically onto a lattice in $G_{0} / K$. So $\Lambda_{0}$ embeds as a discrete subgroup into the isometry group of a negatively curved symmetric space, and we get a contradiction because by the proof of (13) $\Lambda_{0}$ is not isomorphic to the fundamental group of a complete negatively pinched manifold. Thus we are in Case (i), and the claim follows from the fact that $\pi_{1}(N)$ is torsion free and the remarks made after the statement of Theorem 1.4, which are justified in the proof of Theorem 1.4.

Remark 5.3. Of course, if $\operatorname{dim}(M) \leq 3$, then $M \backslash S$ carries a complete finite volume real hyperbolic metric: the 2-dimensional case is classical, and the 3 dimensional case follows from Thurston's hyperbolization theorem. Thus the assumption $n>3$ is needed in (13), (14).

\section{Mostow-type Rigidity FOR HYPERPlane COMPLEMENTS}

Theorem 1.2 follows from Mostow-Prasad rigidity once it is shown that any homotopy equivalence $M_{1} \backslash S_{1} \rightarrow M_{2} \backslash S_{2}$ takes ends of $M_{1} \backslash S_{1}$ to ends of $M_{2} \backslash S_{2}$, which is ensured by part (1) of Theorem 1.1 together with an observation 
that the conjugacy classes of peripheral subgroups of $\pi_{1}(N)$ are permuted by automorphisms of $\pi_{1}(N)$. Details are below.

Proof of Theorem 1.2. We first give a proof when $M_{1}, M_{2}$ are compact, and later indicate modifications in the finite volume case. Let $N_{i}$ denote the complement in $M_{i}$ of an open $\epsilon$-tubular neighborhood of $S_{i}$ where $\epsilon$ is smaller than the normal injectivity radius of $S_{i}$. Contracting along along the geodesic normal to $S_{i}$ identifies $M_{i} \backslash S_{i}$ with the interior of $N_{i}$.

Throughout the proof we suppress the basepoints while talking about fundamental groups. All fundamental groups are taken at some basepoints $p_{i} \in N_{i}$ with $f\left(p_{1}\right)=p_{2}$, and while talking about the fundamental group of a component of $\partial N_{i}$, we implicitly fix an embedded path from $p_{i}$ to each component of $\partial N_{i}$ and really are talking of the union of the component and the path. The same comment applies to components of $S_{i}$.

By Section 4 the group $\pi_{1}\left(N_{i}\right)$ is hyperbolic relative to the fundamental groups of the components of $\partial N_{i}$. A group is called intrinsically elementary if is not isomorphic to a non-elementary subgroup of a relatively hyperbolic group. As noted in [Bel07, discussion after Theorem 1.5]), examples of intrinsically elementary groups include amenable groups and groups with infinite amenable normal subgroups. Since fundamental groups of the components of $\partial N_{i}$ are intrinsically elementary, and since $N_{i}$ is aspherical with incompressible boundary, by a standard argument (spelled out e.g. in [Bel07, Proof of Theorem 8.1]) $f$ induces a homotopy equivalence of pairs $\left(N_{1}, \partial N_{1}\right) \rightarrow\left(N_{2}, \partial N_{2}\right)$ whose pre/postcomposition with homeomorphisms $M_{i} \backslash S_{i} \rightarrow \operatorname{Int}\left(N_{i}\right)$ is homotopic to $f$. With a slight abuse of notations we henceforth denote the homotopy equivalence of pairs by $f$. We can also assume that in each component of $\partial N_{1}$ the map $f:\left(N_{1}, \partial N_{1}\right) \rightarrow\left(N_{2}, \partial N_{2}\right)$ takes homeomorphically one fiber circle to a fiber circle in the corresponding components of $N_{2}$ (because fiber circles correspond to maximal infinite cyclic normal subgroup which are preserved by any group isomorphism).

Let $N_{i}^{d}$ be the union of $N_{i}$ and a collection of 2-discs, one for each component of $\partial N_{i}$, where each disc is attached along the fiber circle in the component of $\partial N_{i}$, and where we may assume that these disks are fibers in disk bundles bounding the chosen circles. Then $f$ extends to a map $f^{d}: N_{1}^{d} \rightarrow N_{2}^{d}$ where $f^{d}$ is a homeomorphism on the corresponding discs. The inclusion $N_{i} \rightarrow M_{i}$ 
extends to an embedding $N_{i}^{d} \rightarrow M_{i}$ which induces, by Van Kampen theorem, an isomorphism of fundamental groups $\pi_{1}\left(N_{i}^{d}\right) \cong \pi_{1}\left(M_{i}\right)$. Then $f^{d}$ induces an isomorphism $\psi: \pi_{1}\left(M_{1}\right) \rightarrow \pi_{1}\left(M_{2}\right)$, and a homotopy equivalence $M_{1} \rightarrow M_{2}$ that extends $f$ up to homotopy. Since under the inclusions $N_{i}^{d} \rightarrow M_{i}$ the fundamental group of any component of $\partial N_{i}$ is mapped onto the corresponding component of $S_{i}$, we conclude that $\psi$ takes the fundamental group of components of $S_{1}$ to the fundamental groups of corresponding components of $S_{2}$. Fix an arbitrary homotopy equivalence $\check{f}: S_{1} \rightarrow S_{2}$ induced by $\psi$; like any homotopy equivalence of closed manifolds $\check{f}$ is onto.

Let $h: M_{1} \rightarrow M_{2}$ be the unique isometry induced by $\psi$ via the Mostow rigidity. Suppressing inclusions, we think of $h, \check{f}$ as maps from $S_{1}$ to $M_{2}$. These maps are homotopic because they are induced by $\psi$, so let $H_{t}: S_{1} \rightarrow M_{2}$ denote the homotopy. The images $h\left(S_{1}\right), \check{f}\left(S_{1}\right)=S_{2}$ are totally geodesic embedded submanifolds of $M_{2}$; we are to show that $h\left(S_{1}\right)=S_{2}$. Fix an arbitrary component $C$ of $S_{1}$, and lift $\left.H_{t}\right|_{C}$ to a homotopy of universal covers $\left.\tilde{H}_{t}\right|_{C}: \tilde{C} \rightarrow \tilde{M}_{2}$ joining two totally geodesic $(n-2)$-planes in $\tilde{M}_{2}=\mathbf{H}^{n}$. By compactness of $C$ there is a uniform upper bound on the lengths of tracks of $\tilde{H}_{t}$, so these totally geodesic $(n-2)$-planes in $\mathbf{H}^{n}$ are within finite Hausdorff distance, hence they coincide, and therefore $h(C)=\check{f}(C)$. Applying this argument to each component of $S_{1}$ gives $h\left(S_{1}\right)=\check{f}\left(S_{1}\right)=S_{2}$.

The uniqueness claim follows from uniqueness in Mostow rigidity. Indeed, assume there are two isometries $h, h^{\prime}$ as above, and let $q:=h^{\prime} \circ h^{-1}$. Then $q$ is an isometry of $M_{2}$ with $q\left(S_{2}\right)=S_{2}$ such that the restriction of $q$ to $M_{2} \backslash S_{2}$ is homotopic to identity. In a smooth manifold any two points can be mapped to each other by an isotopic to identity diffeomorphism, and the isotopy can be chosen equal to identity outside a path joining the points. Compose $q$ with such self-diffeomorphism $r$ of $M_{2}$ so that $r \circ q$ is homotopic to identity relative to a basepoint $p_{2} \in M_{2} \backslash S_{2}$, and $r \circ q=q$ away from a compact set in $M_{2} \backslash S_{2}$. Now take a loop $\gamma$ in $M_{2}$ based at $p_{2}$, and deform it away from $S_{2}$, using that the inclusion $M_{2} \backslash S_{2} \rightarrow M_{2}$ is $\pi_{1}$-surjective. Then $r \circ q$, considered as a self-map of $M_{2} \backslash S_{2}$ takes this loop to a homotopic loop based at $p_{2}$, so $r \circ q$ induces the identity on of $\pi_{1}\left(M_{2}, p_{2}\right)$. Hence $q$ is homotopic to identity, in which case $q$ equals to the identity by Mostow rigidity. 
In the finite volume case we obtain $N_{i}$ by first chopping off the cusps of $M_{i}$ and then removing an $\epsilon$-neighborhood of $S_{i}$, and the rest of the proof is the same.

Proof of Corollary 1.3. immediately follows from Theorem 1.2, where the last assertion of Corollary 1.3 holds as the isometry group of $M$ is finite by Mostow rigidity.

Remark 6.1. Finiteness of Out $\left(\pi_{1}(M \backslash S)\right)$ can be also deduced from part (3) of Theorem 1.1 together with results of Druţu-Sapir [DS08] and the author [Bel07] (see [Bel07, Theorem 1.3] for details).

\section{LATtices that ARE RELATIVELy HyPERBOLIC}

Most of the ideas needed to prove Theorem 1.4 are contained in [ACT02, FW08]. The proof uses structure theory of Lie groups, various basic facts on relatively hyperbolic groups, Margulis's Normal Subgroup Theorem, Osin's result that any relatively hyperbolic group has an infinite normal subgroup of infinite index, and Drutu's result [Dru09] that being relatively hyperbolic is a quasiisometry invariant. Note that Druţu's result is only used to conclude that a group that contains a finite index non-elementary relatively hyperbolic subgroup is non-elementary relatively hyperbolic. It is applied in the "only if" direction of part (i) after we show that the finite index subgroup $\Lambda / \Lambda_{0}$ of $G / G_{0}$ is relatively hyperbolic, and in the "if" direction of part (ii) after we show that the finite index subgroup $\Lambda_{0}$ of $\Lambda$ is relatively hyperbolic. Thus by making the statement of Theorem 1.4 slightly less elegant one could avoid referring to [Dru09]. All the other ingredients of the proof are indispensable.

Remark 7.1. I expect that Theorem 1.4 holds for lattices in algebraic groups over local fields, and encourage an interested reader to work this out. As a starting point, in all cases when the Normal Subgroup Theorem is known e.g. as in [Mar91, BS06], the lattices cannot be relatively hyperbolic. On the other hand, if $k$ is a non-archimedean local field, then all lattices in semisimple algebraic $k$-group of $k$-rank one are relatively hyperbolic, namely, by the proof of [Lub91, Theorem 7.1] every lattice is the fundamental group of a finite graph of group with finite edge groups, and any such group is hyperbolic relative to the vertex subgroups, as follows e.g. from [Bow, Definition 2]. (Nonuniform lattices 
are infinitely generated, but most definitions of relative hyperbolicity extend to infinitely generated case and lead to the same classes of groups [Hru].) My interest in describing lattices that are relatively hyperbolic was motivated by the work of Behrstock-Druţu-Mosher, who by a very different method proved in [BDM09, Section 13] that higher rank lattices in semisimple groups over local fields of characteristic zero are never relatively hyperbolic.

Proof of Theorem 1.4. It is straightforward to check that $\Lambda G_{0}$ is a closed subgroup of $G$, so by $\left[\operatorname{Rag} 72\right.$, Theorem 1.13] the group $\Lambda_{0}:=\Lambda \cap G_{0}$ is a lattice in $G_{0}$. Furthermore, $\Lambda / \Lambda_{0}$ is a lattice in the (discrete) group $G / G_{0}$ because it leaves invariant the pushforward of the $G$-invariant measure on $G / \Lambda$ by the projection $G / \Lambda \rightarrow\left(G / G_{0}\right) /\left(\Lambda / \Lambda_{0}\right)$; in other words $\Lambda / \Lambda_{0}$ is a finite index subgroup of $G / G_{0}$.

Case (i). If $G_{0}$ is compact, then $\Lambda_{0}$ is finite. Therefore, $\Lambda$ is non-elementary relatively hyperbolic if and only if $\Lambda / \Lambda_{0}$ is non-elementary relatively hyperbolic if and only if $G / G_{0}$ is non-elementary relatively hyperbolic (see Appendix A). That relative hyperbolicity of $\Lambda / \Lambda_{0}$ implies relative hyperbolicity of $G / G_{0}$ depends on delicate work of Druţu [Dru09].

Thus it remains to consider the case when $G_{0}$ is noncompact, in which case we are to prove that $\Lambda$ is isomorphic to a relatively hyperbolic group if and only if (ii) holds. First, we treat the "only if" part.

Passing to semisimple quotient. Since $G_{0}$ is noncompact, $\Lambda_{0}$ is infinite. Let $R$ be the solvable radical of $G_{0}$, i.e. a unique maximal solvable connected normal subgroup of $G_{0}$. Note that $R$ is normal in $G$. Let $p: G \rightarrow G / R$ be the projection. Since $\Lambda$ contains no infinite solvable normal subgroup, $R \cap \Lambda$ is finite, and $\Lambda$ is mapped with finite kernel onto a subgroup $p(\Lambda)$ of the Lie group $G / R$ whose identity component $G_{0} / R$ is semisimple.

If $p(\Lambda)$ is not discrete in $G / R$, then by a result of Auslander [Rag72, Theorem 8.24] the identity component $U$ of the closure of $p(\Lambda)$ is solvable. Then $p^{-1}(U)$ is a solvable group that has infinite intersection with $\Lambda$, and it is easy to check that the subgroup $\Lambda \cap p^{-1}(U)$ is normal in $\Lambda$, so this case cannot occur, as $\Lambda$ has no nontrivial solvable normal subgroups and $p^{-1}(U)$ is solvable.

Thus $p(\Lambda)$ discrete. Then it is straightforward to check that $R \Lambda$ is a closed subgroup of $G$, so by [Rag72, Theorem 1.13] the trivial group $R \cap \Lambda$ is a lattice 
in $R$, so $R$ is compact. Recall that a Lie group homomorphism with compact kernel takes lattices to lattices, as can be deduced e.g. from [Rag72, Lemma 1.6], so $p(\Lambda)$ is a lattice in $G / R$, whose identity component $G_{0} / R$ is semisimple.

Passing to centerless semisimple quotient with no compact factors. Let $C$ be a unique maximal connected compact normal subgroup of $G_{0} / R$ so that $H_{0}:=\left(G_{0} / R\right) / C$ is semisimple with no compact factors. By uniqueness, $C$ is normal in $G / R$; we let $H:=(G / R) / C$ and note that $H_{0}$ is the identity component of $H$ as $R, C$ are connected. Since $R$ and $C$ are compact, the kernel of the projection $h: G \rightarrow H$ is compact, so the kernel of $\Lambda \rightarrow h(\Lambda)$ is finite, $h(\Lambda)$ is a lattice in $H$, and $h\left(\Lambda_{0}\right)$ is a lattice in $H_{0}$. In particular, $h(L)$ is still isomorphic to a non-elementary relatively hyperbolic group.

The center $Z$ of $H_{0}$ is normal in $H$. By [Mar91, Lemma IX.6.1], $Z \cap q\left(\Lambda_{0}\right)$ has finite index in $Z$. On the other hand, $Z \cap h(\Lambda)$ is an abelian normal subgroup of $h(\Lambda)$, so $Z \cap h(\Lambda)$ is finite. Thus $Z$ is finite. We let $Q:=H / Z$ and $Q_{0}:=H_{0} / Z$, so that $Q_{0}$ is the identity component of $Q$, and $Q_{0}$ is noncompact semisimple with trivial center and no compact factors. Let $K$ be the (compact) kernel of the projection $q: G \rightarrow Q$; thus the kernel of $\Lambda \rightarrow q(\Lambda)$ is finite (and lies in $G_{0}$ and hence in $\left.\Lambda_{0}\right), \Gamma:=q(\Lambda)$ is a lattice in $Q$, and $\Gamma_{0}:=q\left(\Lambda_{0}\right)$ is a lattice in $Q_{0}$, while $\Gamma$ is still isomorphic to a non-elementary relatively hyperbolic group.

Proving that $G / G_{0}$ is finite. Since the kernel of $q: G \rightarrow Q$ lies in $G_{0}$, it induces an isomorphism $G / G_{0} \rightarrow Q / Q_{0}$ which takes $\Lambda / \Lambda_{0}$ onto $\Gamma / \Gamma_{0}$. As mentioned above, $\Lambda / \Lambda_{0}$ is a lattice in $G / G_{0}$, so $\Gamma / \Gamma_{0}$ is a lattice in $Q / Q_{0}$, which is discrete, and hence it suffices to show that $\Gamma / \Gamma_{0}$ is finite.

Arguing by contradiction suppose that $\Gamma / \Gamma_{0}$ is infinite. The center of $\Gamma_{0}$ is finite, else $\Gamma$ would have an abelian infinite normal subgroup. In fact, it follows from Borel Density theorem that any finite normal subgroup of a lattice in a semisimple Lie group with finite center and no compact factors lies in the center of the Lie group (see e.g. [Wit, Corollary 5.42]). Thus $\Gamma_{0}$ is centerless. Hence the extension

$$
1 \rightarrow \Gamma_{0} \rightarrow \Gamma \rightarrow \Gamma / \Gamma_{0} \rightarrow 1
$$

is completely determined by the canonical homomorphism $\Gamma / \Gamma_{0} \rightarrow \operatorname{Out}\left(\Gamma_{0}\right)$, see [Bro94, IV, Corollary 6.8]. The homomorphism comes from the $\Gamma$-action on $Q_{0}$ by conjugation. Since $Q_{0}$ is semisimple and centerless, $\operatorname{Out}\left(Q_{0}\right)$ is finite [Hel01, Theorem IX.5.4]. Thus after passing to a finite index subgroup, 
we can assume that $\Gamma$ acts on $Q_{0}$ by conjugation via inner automorphisms of $Q_{0}$. Since the actions preserves $\Gamma_{0}$, the homomorphism $\Gamma / \Gamma_{0} \rightarrow \operatorname{Out}\left(\Gamma_{0}\right)$ factors through $N_{Q_{0}}\left(\Gamma_{0}\right) / \Gamma_{0}$, where $N_{Q_{0}}\left(\Gamma_{0}\right)$ denotes the normalizer of $\Gamma_{0}$ in $Q_{0}$. By the Borel Density Theorem, $N_{Q_{0}}\left(\Gamma_{0}\right) / \Gamma_{0}$ is finite (see e.g. [Wit, Corollary 5.43]). Hence after passing to a finite index subgroup we can assume that the homomorphism $\Gamma / \Gamma_{0} \rightarrow \operatorname{Out}\left(\Gamma_{0}\right)$ is trivial, hence a finite index subgroup of $\Gamma$ is the product of two groups, commensurable to $\Gamma_{0}$ and $\Gamma / \Gamma_{0}$, which are infinite. This is impossible for a non-elementary relatively hyperbolic group, which gives a promised contradiction.

$G_{0} / K$ has real rank one. Note that $\Gamma_{0}$ is relatively hyperbolic, as a finite index subgroup of a relatively hyperbolic group $\Gamma$. So $\Gamma_{0}$ is not virtually a product of infinite groups, hence $\Gamma_{0}$ is an irreducible lattice in $Q_{0}$. By Proposition A.2, which is due to Osin, $\Gamma_{0}$ has an infinite normal subgroup of infinite index, which is impossible for higher rank lattices by Margulis's Normal Subgroup Theorem [Mar91, Theorem IX.6.14]. Thus $Q_{0}=G_{0} / K$ has $\mathbb{R}$-rank one.

If (ii) holds, then $\Lambda$ is non-elementary relatively hyperbolic. The projection $G_{0} \rightarrow G_{0} / K$ restricted to $\Lambda_{0}$ has finite kernel and the image is the lattice $\Gamma_{0}$ in the isometry group of a hyperbolic space over reals, quaternions, complex or Cayley numbers. It is well-known that $\Gamma_{0}$ is (non-elementary) relatively hyperbolic, and hence so is $\Lambda_{0}$. By [Dru09] $\Lambda$ is (non-elementary) relatively hyperbolic.

\section{Appendix A. On RELATIVELY hyperbolic GROUPS}

Relatively hyperbolic groups were introduced by Gromov [Gro87], and in this paper we use the following version of Gromov's definition developed by Bowditch (see [Bow, Definition 1]).

Let $G$ be a finitely generated group with a (possibly empty) family $\mathcal{G}$ of infinite finitely generated subgroups. Suppose that $G$ acts properly discontinuously and isometrically on a proper, geodesic, hyperbolic metric space $X$. Then $G$ acts on the ideal boundary $\partial X$ of $X$ as a convergence group [Bow99, Proposition 1.12] with limit set $L(G) \subseteq \partial X$. We refer to [Bow, Section 6], or [Bow99], or [Yam04, Section 5] or [Fre97] for relevant background on convergence groups. 
Definition A.1. We say that $G$ is hyperbolic relative to $\mathcal{G}$ if $L(G)=\partial X$, each point of $L(G)$ is either conical or bounded parabolic, and the maximal parabolic subgroups of $G$ are precisely the elements of $\mathcal{G}$.

Elements of $\mathcal{G}$ are also called peripheral subgroups. It is known that there only finitely many conjugacy classes of peripheral subgroups.

Other definitions of relatively hyperbolic groups were developed by Farb [Far98], Bowditch [Bow, Definition 2], Yaman [Yam04], Druţu-Osin-Sapir [DS05b], Osin [Osi06b], Druţu [Dru09], and Mineyev-Yaman [MY]. It is known that all these definitions are equivalent to Definition A.1, provided $G$ and all its peripheral subgroups are finitely generated and infinite. The proofs of various equivalences can be found in [Dah03, Appendix A] (cf. [Bow, Szc98, Bum05]), [Osi06b, Theorem 7.10], [Yam04], [DS05b, Theorem 8.5], [Dru09, Theorems 4.21,4.34], [MY, Theorem 57]. With many different approaches to relative hyperbolicity there are some minor differences in terminology, which will be pointed out as needed.

Bowditch in [Bow] developed a notion of a relatively hyperbolic boundary for $G$ that is $G$-equivariantly homeomorphic to $\partial X$, and such that any isomorphism of relatively hyperbolic groups that preserves the collection of peripheral subgroups induces an equivariant homeomorphism of the boundaries.

We summarize below some basic properties of (discrete) convergence groups. A subgroup of a convergence group is called elementary if its limit set contains at most two points, which happens exactly if the subgroup is finite, virtually- $\mathbb{Z}$, or parabolic. Otherwise, the subgroup is called non-elementary. An elementary subgroup containing a parabolic element cannot contain a hyperbolic element. Suppose $H$ is a non-elementary convergence group. By a ping-pong argument a non-elementary subgroup of $H$ contains a nonabelian free group [Tuk94]. Any finite normal subgroup of $H$ acts trivially on the limit set, and the limit set of an infinite normal subgroup of $H$ equals to the limit set of $H$ (see [Fre97]). In particular, any infinite normal subgroup of $H$ is non-elementary, and $H$ has no infinite virtually solvable normal subgroups. It follows that $H$ is not virtually a product of infinite groups, for if $H_{1} \times H_{2}$ is a finite index subgroup of $H$ and $H_{1}, H_{2}$ are infinite, then $H_{1} \times H_{2}$, and hence $H_{1}, H_{2}$ are non-elementary, but the normalizer of any infinite order element of $H_{1}$ contains $H_{2}$, and elementary subgroups have elementary normalizers. 
Relative hyperbolicity of $G$ is clearly inherited by extensions $\hat{G}$ of $G$ such that $\hat{G} \rightarrow G$ has finite kernel, and subgroups $\check{G}$ of $G$ of finite index. Indeed, one can use the actions of $\hat{G}, \check{G}$ on the same hyperbolic space induced by the $G$-action, and define the peripheral subgroups of $\hat{G}, \check{G}$ to be stabilizers of the parabolic fixed point of $G$. Also if $N$ is a finite normal subgroup of $G$, then $G / N$ is hyperbolic relative to the images of the peripheral subgroups of $G$, because relative hyperbolicity is encoded in the action on the boundary, and the $G$-action on the boundary factors through the projection $G \rightarrow G / N$ (an algebraic proof of this fact can be found in [AMO07, Lemma 4.4]). It follows that the kernel of the action of a relatively hyperbolic group on its boundary is a unique maximal normal finite subgroup, which was characterized in purely group theoretic terms in [AMO07, Lemma 3.3]. Finally, if $G$ is non-elementary, then $\hat{G}, \check{G}, G / N$ are non-elementary because all these groups share the same boundary.

By contrast, is not at all easy to show that relative hyperbolicity is inherited by any group $\tilde{G}$ that contains $G$ as a finite index subgroup. Druţu recently proved [Dru09] a much more general result that the property of being non-elementary relatively hyperbolic is invariant under quasi-isometry.

The following result does not appear in the literature.

Proposition A.2. (Osin) Any non-elementary relatively hyperbolic group has an infinite normal subgroup of infinite index.

Proof. The proof below applies to all groups that are relatively hyperbolic in the sense of Osin, and there are various differences in terminology, e.g. Osin reserves the term "elementary" for virtually cyclic subgroups, and calls an element "hyperbolic" if it cannot be conjugated into a peripheral subgroup; an element of infinite order is hyperbolic in Osin's sense if and only if it fixes exactly two points on the boundary. In what follows we rephrase Osin's results in the terminology adopted in this paper.

Take a non-elementary relatively hyperbolic group $G$ with peripheral subgroups $H_{\lambda}$. By [Osi06a, Corollary 4.5] $G$ contains an (infinite order) hyperbolic element. By [Osi06a, Theorem 4.3] every (infinite order) hyperbolic element $g$ is contained in a unique maximal virtually cyclic subgroup $E(g)$. By [Osi06a, Corollary 1.7] $G$ is hyperbolic relative to $\left\{H_{\lambda}, E(g)\right\}$ which is non-elementary, 
since the original relatively hyperbolic structure on $G$ was non-elementary. Repeating this argument we find another (infinite order) hyperbolic element $h$ such that $G$ is hyperbolic to $\left\{H_{\lambda}, E(g), E(h)\right\}$. By [Osi07, Theorem 1.1] for all large $n$ the quotient $\bar{G}$ of $G$ by the relation $h^{n}=1$ is hyperbolic relative to the images of $\left\{H_{\lambda}, E(g), E(h)\right\}$ and the projection map $G \rightarrow \bar{G}$ is one-to-one on each peripheral subgroups other than $E(h)$. In particular, the group $\bar{G}$ is infinite, as it contains the infinite virtually cyclic subgroup $E(g)$ (and in fact one can easily show that $\bar{G}$ is non-elementary, as at the end of the proof of [Osi07, Corollary 1.6]). The kernel is also infinite because it contains the infinite cyclic subgroup generated by $h^{n}$, thus the kernel is an infinite normal subgroup of infinite index.

\section{Appendix B. Morse theOretic lemma}

Particular cases of the following lemma were used by Toledo [Tol93] and also by those working with 3-hyperbolic manifolds (as explained to me by Ian Agol).

Suppose that $M$ is a connected complete $n$-manifold of $\sec \leq 0$, and $S$ is a compact (not necessarily connected) totally geodesic submanifold of $M$ of codimension $>1$. Denote the universal cover of $M$ by $\tilde{M}$, and let $\tilde{S}$ be the preimage of $S$ under $\tilde{M} \rightarrow M$. Let $N$ be the complement in $M$ of an open tubular neighborhood of $S$.

Lemma B.1. (i) $\tilde{M} \backslash \tilde{S}$ is diffeomorphic to the manifold obtained from an open $n$-ball by attaching $k$-handles, one for each component of $\tilde{S}$ of codimension $k+1$. (ii) $M \backslash S$ is aspherical if and only if each component of $S$ has codimension two, in which case the inclusion $M \backslash S \rightarrow M$ induces a surjection $\pi_{1}(M \backslash S) \rightarrow \pi_{1}(M)$ whose kernel is a free group on the set of components of $\tilde{S}$.

(iii) no homotopically nontrivial loop in $\partial N$ is null-homotopic in $N$.

Proof. Since $S$ is compact it has positive injectivity radius $i$. Let $Z$ be the complement of an open $i$-neighborhood of $\tilde{S}$ in $\tilde{M}$. Fix a point $*$ in the interior of $Z$. The distance to $*$ is a smooth Morse function on $Z$. Indeed, $\operatorname{since} \sec (\tilde{M}) \leq$ 0 , the function has no critical points on the interior of $Z$. By first variation formula the critical points of the function are the points where geodesic rays emanating from $*$ intersect $\partial Z$ orthogonally. Thus if $C$ is a component of $\tilde{S}$, then the $i$-neighborhood of $C$ contains precisely two critical points which lie on 
a unique ray from $*$ that is orthogonal to $C$. By Morse theory passing through $C$ results in attaching a handle that "goes around" $C$, thus if $C$ has codimension $k+1$ we attach a $k$-handle, so (i) is proved.

(ii) Since $S$ has codimension $>1$, all $k$ 's are positive, so that $Z$ is connected and homotopy equivalent to a wedge of spheres with one $k$-sphere for each $k$ handle "going around" a component of $\partial Z$. Hence $Z$ is aspherical if and only if $Z$ is homotopy equivalent to a wedge of circles, or equivalently if and only if each component of $S$ has codimension two. In this case the inclusion $M \backslash S \rightarrow M$ clearly induces a surjection $\pi_{1}(M \backslash S) \rightarrow \pi_{1}(M)$ whose kernel $\pi_{1}(Z)$ is isomorphic to a free group on the set of components of $\partial Z$.

(iii) If a loop $\alpha \subset \partial N$ is null-homotopic in $N$, then $\alpha$ lifts to a loop $\tilde{\alpha} \subset \partial Z$ that is null-homotopic in $Z$. Every component of $\partial Z$ is homotopy equivalent to a circle that represents a nontrivial free generator in $\pi_{1}(Z)$. Thus $\tilde{\alpha}$ must be null-homotopic in $\partial Z$, and this homotopy can be pushed down to $\partial N$.

\section{Appendix C. Curvature of Warped product metrics}

In this appendix we review (and also correct!) some formulas for the curvature tensor of a multiply-warped product metric $d r^{2}+g_{r}$ that were worked out in [BW04, Section 6].

Suppose $g_{r}$ is a family of metric on a manifold $F$ where $r$ is on an open interval $I$. The computation in [BW04, Section 6]) works provided at each point $w$ of $F$ there is a basis of vector fields $\left\{X_{i}\right\}$ on a neighborhood $U_{w} \subset F$ that is $g_{r}$-orthogonal for each $r$. We fix one such a basis for each $w$. Let $h_{i}(r)=$ $\sqrt{g_{r}\left(X_{i}, X_{i}\right)}$ so that $Y_{i}=X_{i} / h_{i}$ form a $g_{r}$-orthonormal basis on $U_{w}$ for any $r>0$. Since $X_{i} \neq 0$ and $g_{r}$ is nondegenerate, $h_{i}>0$

To simplify some of the formulas below we denote $g(X, Y)$ by $\langle X, Y\rangle$, denote the vector field $\frac{\partial}{\partial r}$ by $\partial_{r}$, and reserve the notation $\frac{\partial}{\partial r} T$ for the partial derivative of the function $T$ by $r$. 
A straightforward tedious computation (done e.g. in [BW04, Section 6]) yields the following.

$$
\begin{gathered}
\left\langle R_{g}\left(Y_{i}, Y_{j}\right) Y_{j}, Y_{i}\right\rangle=\left\langle R_{g_{r}}\left(Y_{i}, Y_{j}\right) Y_{j}, Y_{i}\right\rangle-\frac{h_{i}^{\prime} h_{j}^{\prime}}{h_{i} h_{j}}, \\
\left\langle R_{g}\left(Y_{i}, Y_{j}\right) Y_{l}, Y_{m}\right\rangle=\left\langle R_{g_{r}}\left(Y_{i}, Y_{j}\right) Y_{l}, Y_{m}\right\rangle \quad \text { if }\{i, j\} \neq\{l, m\}, \\
\left.\left.\left\langle R_{g}\left(Y_{i}, \partial_{r}\right) \partial_{r}\right), Y_{i}\right\rangle=-\frac{h_{i}^{\prime \prime}}{h_{i}}, \quad\left\langle R_{g}\left(Y_{i}, \partial_{r}\right) \partial_{r}\right), Y_{j}\right\rangle=0 \quad \text { if } i \neq j .
\end{gathered}
$$

The following mixed term is by far the most complicated and is usually the hardest to control.

Lemma C.4. $2\left\langle R_{g}\left(\partial_{r}, Y_{i}\right) Y_{j}, Y_{k}\right\rangle$ equals to

$$
\left\langle\left[Y_{i}, Y_{j}\right], Y_{k}\right\rangle\left(\ln \frac{h_{k}}{h_{j}}\right)^{\prime}+\left\langle\left[Y_{k}, Y_{i}\right], Y_{j}\right\rangle\left(\ln \frac{h_{j}}{h_{k}}\right)^{\prime}+\left\langle\left[Y_{k}, Y_{j}\right], Y_{i}\right\rangle\left(\ln \frac{h_{i}^{2}}{h_{j} h_{k}}\right)^{\prime} .
$$

Proof. As noted in [BW04, Section 6] $\nabla_{\partial_{r}} Y_{i}=0$ and $\left[\partial_{r}, Y_{i}\right]=-\frac{h_{i}^{\prime}}{h_{i}} Y_{i}$, which by the definition of the curvature tensor gives that

$$
R\left(\partial_{r}, Y_{i}\right) Y_{j}=\nabla_{\partial_{r}} \nabla_{Y_{i}} Y_{j}+\frac{h_{i}^{\prime}}{h_{i}} \nabla_{Y_{i}} Y_{j}
$$

Just like in [BW04] Koszul's formula can be used to compute that

$$
\begin{gathered}
\nabla_{Y_{i}} Y_{j}=-\frac{h_{i}^{\prime}}{h_{i}} \delta_{i j} \partial_{r}+\sum_{k} \frac{Q_{i j k}}{2} Y_{k} \quad \text { where } \\
Q_{i j k}=\left\langle\left[Y_{i}, Y_{j}\right], Y_{k}\right\rangle+\left\langle\left[Y_{k}, Y_{i}\right], Y_{j}\right\rangle+\left\langle\left[Y_{k}, Y_{j}\right], Y_{i}\right\rangle .
\end{gathered}
$$

and then the definition of the curvature tensor implies that

$$
2\left\langle R_{g}\left(\partial_{r}, Y_{i}\right) Y_{j}, Y_{k}\right\rangle=\frac{h_{i}^{\prime}}{h_{i}} Q_{i j k}+\frac{\partial}{\partial r} Q_{i j k} .
$$

Since $\nabla_{\partial_{r}} Y_{k}=0$, we know that $\frac{\partial}{\partial r} Q_{i j k}$ is the sum of terms of the form $\left\langle\nabla_{\partial_{r}}\left[Y_{i}, Y_{j}\right], Y_{k}\right\rangle$. It is noted in [BW04, Section 6] that $\left[Y_{i}, Y_{j}\right]=\frac{1}{h_{i} h_{j}}\left[X_{i}, X_{j}\right]$ so it is enough to compute $\nabla_{\partial_{r}}\left[X_{i}, X_{j}\right]$. We can write $\left[X_{i}, X_{j}\right]=\sum_{k} c_{i j}^{k} X_{k}$, where $c_{i j}^{k}$ is independent of $r$ (because the identity $\left[X_{i}, X_{j}\right]=\sum_{k} c_{i j}^{k} X_{k}$ is pullbacked from $T F$ by the projection $F \times I \rightarrow F)$. Thus $\frac{\partial}{\partial r} c_{i j}^{k}=0$, yet one has $c_{i j}^{k}=\left\langle\left[X_{i}, X_{j}\right], X_{k}\right\rangle / h_{k}^{2}$ because $\left\langle X_{k}, X_{k}\right\rangle=h_{k}^{2}$. On the other hand, $\nabla_{\partial_{r}} Y_{k}=0$ implies that $\nabla_{\partial_{r}} X_{k}=\frac{h_{k}^{\prime}}{h_{k}} X_{k}$, and in summary $\nabla_{\partial_{r}}\left[X_{i}, X_{j}\right]=\sum_{k} c_{i j}^{k} \nabla_{\partial_{r}} X_{k}$, where

$$
c_{i j}^{k} \nabla_{\partial_{r}} X_{k}=c_{i j}^{k} \frac{h_{k}^{\prime}}{h_{k}} X_{k}=\left\langle\left[X_{i}, X_{j}\right], X_{k}\right\rangle \frac{h_{k}^{\prime}}{h_{k}^{3}} X_{k}=\left\langle\left[Y_{i}, Y_{j}\right], Y_{k}\right\rangle \frac{h_{k}^{\prime}}{h_{k}} h_{i} h_{j} Y_{k},
$$


which yields

$$
\begin{gathered}
\left\langle\nabla_{\partial_{r}}\left[Y_{i}, Y_{j}\right], Y_{k}\right\rangle=\left\langle\frac{1}{h_{i} h_{j}} \nabla_{\partial_{r}}\left[X_{i}, X_{j}\right]+\left(\frac{1}{h_{i} h_{j}}\right)^{\prime}\left[X_{i}, X_{j}\right], Y_{k}\right\rangle= \\
\left(\frac{h_{k}^{\prime}}{h_{k}}-\frac{h_{i}^{\prime} h_{j}^{\prime}}{h_{i} h_{j}}\right)\left\langle\left[Y_{i}, Y_{j}\right], Y_{k}\right\rangle=\left(\ln \frac{h_{k}}{h_{i} h_{j}}\right)^{\prime}\left\langle\left[Y_{i}, Y_{j}\right], Y_{k}\right\rangle,
\end{gathered}
$$

giving the desired formula, after plugging (C.7) into (C.6) and collecting terms.

Remark C.8. It was stated in [BK05, BK06] as an immediate implication of [BW04, Section 6] that

$$
\left\langle R_{g}\left(\partial_{r}, Y_{i}\right) Y_{j}, Y_{k}\right\rangle=\frac{\left(\ln \left(h_{j} h_{k}\right)\right)^{\prime}}{2}\left(\left\langle\left[Y_{j}, Y_{i}\right], Y_{k}\right\rangle+\left\langle\left[Y_{i}, Y_{k}\right], Y_{j}\right\rangle+\left\langle\left[Y_{j}, Y_{k}\right], Y_{i}\right\rangle\right),
$$

but according to Lemma C.4, this formula is incorrect due to error in [BW04, Lemma 6.2(1)]. (Note that [BW04, Lemma 6.2(4)] is also incorrect as a particular case of [BW04, Lemma 6.2(1)]). This erroneous formula was used in [BW04, BK05, BK06] in a crucial way, yet all the other results in [BW04, BK05, BK06] continue to hold without any change with the correct formula of Lemma C.4, because they only depend on the fact that $\left\langle R_{g}\left(\partial_{r}, Y_{i}\right) Y_{j}, Y_{k}\right\rangle$ is a linear combination of the terms of the form $\frac{h_{l}^{\prime}}{h_{l}}\left\langle\left[Y_{i}, Y_{j}\right], Y_{k}\right\rangle$. Specifically, in [BW04] we always work in the frame $\left\{Y_{k}\right\}$ for which $\left\langle\left[Y_{j}, Y_{i}\right], Y_{k}\right\rangle$ vanishes at the point where the curvature is computed (see [BW04, Section 7]), so $\left\langle R_{g}\left(\partial_{r}, Y_{i}\right) Y_{j}, Y_{k}\right\rangle$ also vanishes at the point. Similarly, in the nearly identical warped product computations of [BK05, Section 3], and [BK06, Section 10] the terms $\frac{h_{l}^{\prime}}{h_{l}}$ are uniformly bounded, and we work with a family of metrics $d r^{2}+s^{2} g_{r}$ for which $\left\langle\left[Y_{j}, Y_{i}\right], Y_{k}\right\rangle$ approaches zero as $s \rightarrow \infty$, so $\left\langle R_{g}\left(\partial_{r}, Y_{i}\right) Y_{j}, Y_{k}\right\rangle$ is asymptotically zero, which is what is needed in $[\mathrm{BK} 05, \mathrm{BK} 06]$.

\section{Appendix D. Another proof of RELATIVe hyperbolicity}

This appendix gives another proof of part (1) of Theorem 1.1 via results of Heintze, Schroeder [Sch91], Kapovich-Leeb [KL95] and Druţu-Osin-Sapir [DS05b].

We refer to [DS05b] for basic information on asymptotic cones and tree-graded spaces.

Suppose that $X$ is a $C A T(0)$ space with a collection $\left\{D_{i}\right\}$ of open convex subspaces of $X$ such that there exists $\epsilon>0$ with the property that $\epsilon$-neighborhoods of $D_{i}$ 's are disjoint, and every $2 \epsilon$-ball centered outside $\cup_{i} D_{i}$ is $C A T(-1)$. Fix an 
arbitrary asymptotic cone Cone $(X)$ of $X$ (specified by a choice of non-principal ultrafilter, an observation point, and a sequence of scaling factors), and denote by $\mathcal{D}$ the collection of all subspaces of $\operatorname{Cone}(X)$ that are limits of various subsequences in $\left\{D_{i}\right\}$. Suppose that every subspace $D \in \mathcal{D}$ has the following property: given any distinct points $x, y, z \in \mathcal{D}$ there is a sequence $z_{n} \rightarrow z$ such that the geodesic triangle with vertices $x, y, z_{n}$ is open (where following Kapovich-Leeb we call a geodesic triangle open if any two sides of the triangle intersect only at their common vertex). Suppose that a group $G$ acts geometrically (i.e. isometrically, properly discontinuously, and cocompactly) on $X$, and that the action permutes $D_{i}$ 's. With the above assumptions one has the following.

Theorem D.1. $G$ is hyperbolic relative to the set of stabilizers of $D_{i}$ 's.

Proof of Theorem D.1. In [KL95, Section 4.2] it is proved that Cone $(X)$ is treegraded with respect to $\mathcal{D}$. (The result does not need the existence of a geometric action of $G$ on $X$ that permutes $D_{i}$ 's, and is stated in [KL95] only when $D_{i}$ 's are uniform neighborhood of flats, in which case each $D$ is a flat in $\operatorname{Cone}(X)$, but the proof in [KL95] works without change).

Since $\epsilon$-neighborhoods of $D_{i}$ 's are disjoint and $G$ acts geometrically, $\left\{D_{i}\right\}$ falls into finitely many $G$-orbits. (Else there would exist an infinite sequence of pairwise $G$-inequivalent $D_{k}$ 's, so take a point in each, pass to quotient, choose a converging subsequence, lift it to $X$, and conclude that after changing $D_{k}$ 's within their $G$-orbits, we can assume that they intersect an arbitrary small ball around some point of $X$, contradicting that $D_{i}$ 's are $\epsilon$-separated). Pick a representative in each orbit and denote the representatives by $D_{i_{1}}, \ldots, D_{i_{k}}$. Denote by $H_{j}$ the stabilizer of $D_{i_{j}}$ in $G$.

The $H_{j}$-action on the closure of $D_{i_{j}}$ is geometric. (The action is clearly properly discontinuous and isometric, and $D_{i_{j}} / H_{j}$ is precompact, because by construction the inclusion $D_{i_{j}} \rightarrow X$ descends to the inclusion $D_{i_{j}} / H_{j} \rightarrow X / G$, and $X / G$ is compact).

Fix a base point $*$ in $X$, and consider the orbit map $q_{*}: G \rightarrow X$, which is a quasi-isometry for any word metric on $G$. Then there exists $r>0$ such that $q_{*}\left(H_{j}\right)$ is $r$-close to $D_{i_{j}}$ in the Hausdorff topology for each $j=1, \ldots, k$. (For if $*_{j}$ is the nearest point projection of $*$ onto $D_{i_{j}}$, then the $H_{j}$-orbit of $*_{j}$ is 
within finite Hausdorff distance from $D_{i_{j}}$ as well as from $\left.q_{*}\left(H_{j}\right)\right)$. Therefore, $q_{*}\left(g H_{j}\right)$ and $g\left(D_{i_{j}}\right)$ are $r$-close in the Hausdorff topology.

Thus the bi-Lipschitz homeomorphism of asymptotic cones Cone $(G) \rightarrow \operatorname{Cone}(X)$ induced by $q_{*}$ maps limits of sequences of left cosets of $H_{j}$ 's homeomorphically onto the corresponding elements of $\mathcal{D}$, which are pieces of the tree-graded space structure on $X$. For geodesic metric spaces the property of being tree-graded is a preserved under or homeomorphism [DS05b, Remark 2.18], so Cone $(G)$ is tree-graded with respect to limits of sequences of left cosets of $H_{j}$ 's. By [DS05b, Theorem 1.11] this implies that $G$ is hyperbolic relative to the set of conjugates of $H_{1}, \ldots, H_{k}$, which equals to the set of stabilizers of $D_{i}$ 's in $G$.

Finally, we are ready to give another proof of part (1) of Theorem 1.1. Suppose $M$ is a complete finite volume real hyperbolic $n$-manifold, and $S$ is a compact totally geodesic submanifold of codimension two. As in Remark 3.4 we give $N$ the Heintze-Schroeder metric with sec $\leq 0$ in which $\partial N$ is totally geodesic and sec $<0$ on the interior of $N$. Let $G:=\pi_{1}(N)$ and $X$ be the universal cover of $N$, so that $G$ acts geometrically on the $C A T(0)$ space $X$. Let $\iota$ be the normal injectivity radius of $\partial N$. Fix a positive $\epsilon<\frac{\iota}{4}$, consider the preimage of the $\epsilon+\frac{\iota}{2}$-neighborhood of $\partial N$ under the covering $X \rightarrow N$, and denote its pathcomponents by $D_{i}$ 's. Since $\partial N$ is locally convex and $X$ is $C A T(0)$, each $D_{i}$ is convex. The $\epsilon$-neighborhoods of $D_{i}$ 's are disjoint, and the sectional curvature is negative on any closed $2 \epsilon$-ball centered outside $\cup_{i} D_{i}$, so after rescaling we can assume that each $2 \epsilon$-ball centered outside $\cup_{i} D_{i}$ is $C A T(-1)$. Since $\partial N$ is totally geodesic, it is $\pi_{1}$-incompressible. Denote the subgroups of $G$ corresponding to the fundamental groups of the path-components of $\partial N$ by $H_{1}, \ldots, H_{k}$; the stabilizer of each $D_{i}$ is conjugate to some $H_{j(i)}$. The universal cover of every component of $\partial N$ is isometric to $\mathbb{R}^{n-1}$ or to $\mathbb{R} \times \mathbf{H}^{n-2}$, hence their limits in Cone $(X)$ are isometric to an asymptotic cone of a fixed $D_{i}$, which is either $\mathbb{R}^{n-1}$, or the product of $\mathbb{R}$ and a metric tree T. Applying Lemma D. 2 below, we see that all assumptions of Theorem D.1 hold.

Lemma D.2. If $T$ is a metric tree, then for any geodesic triangle in $\mathbb{R} \times T$ with vertices $x, y, z$ there is a sequence $z_{n} \rightarrow z$ such that the geodesic triangle with vertices $x, y, z_{n}$ is open. 
Proof. Denote the projection of $w$ to the $T$-factor by $\bar{w}$. If $\bar{z}$ lies on a line through $\bar{x}, \bar{y}$, then $x, y, z$ lie on a (flat) plane in $\mathbb{R} \times T$, so we can just pick $z_{n}$ outside the line through $x, y$ in the plane. Otherwise $\bar{z}, \bar{x}, \bar{y}$ are the endpoints of a tripod $\Upsilon$; denote by $\bar{m}$ the point on $\Upsilon$ of valency 3 . The product $\mathbb{R} \times \Upsilon$ is the union of three flat strips joined at the line $\mathbb{R} \times\{\bar{m}\}$; let $m$ be the point where this line intersects the segment $[x, y]$. It suffices to choose $z_{n}$ so that the intersection of $\mathbb{R} \times\{\bar{m}\}$ with the segments $[x, y],\left[x, z_{n}\right],\left[y, z_{n}\right]$ are distinct points, and this can be arranged by choosing $z_{n} \rightarrow z$ outside the straight lines that extend the segments $[x, m],[y, m]$ into the strip containing $z$.

Acknowledgments. This paper is dedicated to Thomas Farrell and Lowell Jones in appreciation of their seminal work on topological rigidity; in fact this project was initiated because I got invited to the 2007 Morelia Conference in honor of Farrell and Jones. It is a pleasure to thank Ian Agol, Ken Baker, Oleg V. Belegradek, David Fisher, Vitali Kapovitch, Denis Osin, and the referees for discussions and communications relevant to this work.

\section{REFERENCES}

$\left[\mathrm{ABC}^{+}\right.$96] J. Amorós, M. Burger, K. Corlette, D. Kotschick, and D. Toledo, Fundamental groups of compact Kähler manifolds, Mathematical Surveys and Monographs, vol. 44, American Mathematical Society, Providence, RI, 1996.

[ACT02] D. Allcock, J. A. Carlson, and D. Toledo, Orthogonal complex hyperbolic arrangements, Symposium in Honor of C. H. Clemens (Salt Lake City, UT, 2000), Contemp. Math., vol. 312, Amer. Math. Soc., Providence, RI, 2002, pp. 1-8.

[AMO07] G. Arzhantseva, A. Minasyan, and D. Osin, The SQ-universality and residual properties of relatively hyperbolic groups, J. Algebra 315 (2007), no. 1, 165-177.

[BD06] G. C. Bell and A. N. Dranishnikov, A Hurewicz-type theorem for asymptotic dimension and applications to geometric group theory, Trans. Amer. Math. Soc. 358 (2006), no. 11, 4749-4764 (electronic).

[BD08] G. Bell and A. Dranishnikov, Asymptotic dimension, Topology Appl. 155 (2008), no. $12,1265-1296$.

[BDM09] J. Behrstock, C. Druţu, and L. Mosher, Thick metric spaces, relative hyperbolicity, and quasi-isometric rigidity, Math. Ann. 344 (2009), no. 3, 543-595.

[Bel] I. Belegradek, Complex hyperbolic hyperplane complements, arXiv:0711.5001v1 [math.GR].

[Bel07] _ Aspherical manifolds with relatively hyperbolic fundamental groups, Geom. Dedicata 129 (2007), 119-144.

[Bes87] A. L. Besse, Einstein manifolds, Ergebnisse der Mathematik und ihrer Grenzgebiete (3), vol. 10, Springer-Verlag, Berlin, 1987. 
[BH99] M. R. Bridson and A. Haefliger, Metric spaces of non-positive curvature, Grundlehren der Mathematischen Wissenschaften, vol. 319, Springer-Verlag, Berlin, 1999.

[BK05] I. Belegradek and V. Kapovitch, Pinching estimates for negatively curved manifolds with nilpotent fundamental groups, Geom. Funct. Anal. 15 (2005), no. 5, 929-938.

[BK06]_ Classification of negatively pinched manifolds with amenable fundamental groups, Acta Math. 196 (2006), no. 2, 229-260.

[Bow] B. H. Bowditch, Relatively hyperbolic groups, Southampton preprint, 1999, http://www.warwick.ac.uk/ masgak/preprints.html.

[Bow93] _ Discrete parabolic groups, J. Differential Geom. 38 (1993), no. 3, 559-583.

[Bow99] Convergence groups and configuration spaces, Geometric group theory down under (Canberra, 1996), de Gruyter, Berlin, 1999, pp. 23-54.

[Bro94] K. S. Brown, Cohomology of groups, Graduate Texts in Mathematics, vol. 87, Springer-Verlag, New York, 1994, Corrected reprint of the 1982 original.

[BS06] U. Bader and Y. Shalom, Factor and normal subgroup theorems for lattices in products of groups, Invent. Math. 163 (2006), no. 2, 415-454.

[Bum05] I. Bumagin, On definitions of relatively hyperbolic groups, Geometric methods in group theory, Contemp. Math., vol. 372, Amer. Math. Soc., Providence, RI, 2005, pp. 189-196.

[BW04] I. Belegradek and G. Wei, Metrics of positive Ricci curvature on bundles, Int. Math. Res. Not. (2004), no. 57, 3079-3096.

[CD95] R. M. Charney and M. W. Davis, Strict hyperbolization, Topology 34 (1995), no. 2, 329-350.

[CT89] J. A. Carlson and D. Toledo, Harmonic mappings of Kähler manifolds to locally symmetric spaces, Inst. Hautes Études Sci. Publ. Math. (1989), no. 69, 173-201.

[Dah03] F. Dahmani, Les groupes relativement hyperboliques et leurs bords, Ph.D. thesis, Université Louis Pasteur, Strasbourg, 2003.

[DJ91] M. W. Davis and T. Januszkiewicz, Hyperbolization of polyhedra, J. Differential Geom. 34 (1991), no. 2, 347-388.

[dlHV89] P. de la Harpe and A. Valette, La propriété $(T)$ de Kazhdan pour les groupes localement compacts (avec un appendice de Marc Burger), Astérisque (1989), no. 175, 158, With an appendix by M. Burger.

[Dru09] C. Druţu, Relatively hyperbolic groups: geometry and quasi-isometric invariance, Comment. Math. Helv. 84 (2009), no. 3, 503-546.

[DS05a] C. Druţu and M. Sapir, Relatively hyperbolic groups with rapid decay property, Int. Math. Res. Not. (2005), no. 19, 1181-1194.

[DS05b] _ Tree-graded spaces and asymptotic cones of groups, Topology 44 (2005), no. 5, 959-1058, With an appendix by Denis Osin and Sapir.

[DS08] C. Druţu and M. V. Sapir, Groups acting on tree-graded spaces and splittings of relatively hyperbolic groups, Adv. Math. 217 (2008), no. 3, 1313-1367. 
$\left[\mathrm{ECH}^{+}\right.$92] D. B. A. Epstein, J. W. Cannon, D. F. Holt, S. V. F. Levy, M. S. Paterson, and W. P. Thurston, Word processing in groups, Jones and Bartlett Publishers, Boston, MA, 1992.

[Far97] B. Farb, The quasi-isometry classification of lattices in semisimple Lie groups, Math. Res. Lett. 4 (1997), no. 5, 705-717.

[Far98] _ Relatively hyperbolic groups, Geom. Funct. Anal. 8 (1998), no. 5, 810-840.

[FJ98] F. T. Farrell and L. E. Jones, Rigidity for aspherical manifolds with $\pi_{1} \subset \mathrm{GL}_{m}(\mathbf{R})$, Asian J. Math. 2 (1998), no. 2, 215-262.

[Fre97] E. M. Freden, Properties of convergence groups and spaces, Conform. Geom. Dyn. 1 (1997), 13-23 (electronic).

[Fuj88] K. Fujiwara, A construction of negatively curved manifolds, Proc. Japan Acad. Ser. A Math. Sci. 64 (1988), no. 9, 352-355.

[FW08] B. Farb and S. Weinberger, Isometries, rigidity and universal covers, Ann. of Math. (2) 168 (2008), no. 3, 915-940.

[GM08] D. Groves and J. F. Manning, Dehn filling in relatively hyperbolic groups, Israel J. Math. 168 (2008), no. 1, 317-429.

[Gro87] M. Gromov, Hyperbolic groups, Essays in group theory, Math. Sci. Res. Inst. Publ., vol. 8, Springer, 1987, pp. 75-263.

[Hel01] S. Helgason, Differential geometry, Lie groups, and symmetric spaces, Graduate Studies in Mathematics, vol. 34, American Mathematical Society, Providence, RI, 2001, Corrected reprint of the 1978 original.

[Hru] G. C. Hruska, Relative hyperbolicity and relative quasiconvexity for countable groups, arXiv:0801.4596v2 [math.GR].

[Jol90] P. Jolissaint, Rapidly decreasing functions in reduced $C^{*}$-algebras of groups, Trans. Amer. Math. Soc. 317 (1990), no. 1, 167-196.

[JY91] J. Jost and S.-T. Yau, Harmonic maps and group representations, Differential geometry, Pitman Monogr. Surveys Pure Appl. Math., vol. 52, Longman Sci. Tech., Harlow, 1991, pp. 241-259.

[KL95] M. Kapovich and B. Leeb, On asymptotic cones and quasi-isometry classes of fundamental groups of 3 -manifolds, Geom. Funct. Anal. 5 (1995), no. 3, 582-603.

[KN63] S. Kobayashi and K. Nomizu, Foundations of differential geometry. Vol I, Interscience Publishers, a division of John Wiley \& Sons, New York-Lond on, 1963.

[Lab91] F. Labourie, Existence d'applications harmoniques tordues à valeurs dans les variétés à courbure négative, Proc. Amer. Math. Soc. 111 (1991), no. 3, 877-882.

[Laf02] V. Lafforgue, $K$-théorie bivariante pour les algèbres de Banach et conjecture de Baum-Connes, Invent. Math. 149 (2002), no. 1, 1-95.

[Lub91] A. Lubotzky, Lattices in rank one Lie groups over local fields, Geom. Funct. Anal. 1 (1991), no. 4, 406-431.

[Mar91] G. A. Margulis, Discrete subgroups of semisimple Lie groups, Ergebnisse der Mathematik und ihrer Grenzgebiete (3), vol. 17, Springer-Verlag, Berlin, 1991.

[MY] I. Mineyev and A. Yaman, Relative hyperbolicity and bounded cohomology, preprint, 2007, http://www.math.uiuc.edu/ mineyev/math/. 
[Nos92] G. A. Noskov, Algebras of rapidly decreasing functions on groups and cocycles of polynomial growth, Sibirsk. Mat. Zh. 33 (1992), no. 4, 97-103, 221.

[NR97] W. D. Neumann and L. Reeves, Central extensions of word hyperbolic groups, Ann. of Math. (2) 145 (1997), no. 1, 183-192.

[Osi05] D. V. Osin, Asymptotic dimension of relatively hyperbolic groups, Int. Math. Res. Not. (2005), no. 35, 2143-2161.

[Osi06a] Elementary subgroups of relatively hyperbolic groups and bounded generation, Internat. J. Algebra Comput. 16 (2006), no. 1, 99-118.

[Osi06b] , Relatively hyperbolic groups: intrinsic geometry, algebraic properties, and algorithmic problems, Mem. Amer. Math. Soc. 179 (2006), no. 843, vi+100.

[Osi07] _ Peripheral fillings of relatively hyperbolic groups, Invent. Math. 167 (2007), no. 2, 295-326. MR MR2270456

[Pet06] P. Petersen, Riemannian geometry, second ed., Graduate Texts in Mathematics, vol. 171, Springer, New York, 2006.

[Rag72] M. S. Raghunathan, Discrete subgroups of Lie groups, Springer-Verlag, New York, 1972, Ergebnisse der Mathematik und ihrer Grenzgebiete, Band 68.

[Rag84] _ Torsion in cocompact lattices in coverings of $\operatorname{Spin}(2, n)$, Math. Ann. 266 (1984), no. 4, 403-419.

[Reb01] D. Y. Rebbechi, Algorithmic properties of relatively hyperbolic groups, Ph.D. thesis, Rutgers Newark, 2001.

[Roe05] J. Roe, Hyperbolic groups have finite asymptotic dimension, Proc. Amer. Math. Soc. 133 (2005), no. 9, 2489-2490 (electronic).

[Sak96] T. Sakai, Riemannian geometry, Translations of Mathematical Monographs, vol. 149, American Mathematical Society, Providence, RI, 1996, Translated from the 1992 Japanese original by the author.

[Sch91] V. Schroeder, Analytic manifolds of nonpositive curvature with higher rank subspaces, Arch. Math. (Basel) 56 (1991), no. 1, 81-85.

[Sel99] Z. Sela, Endomorphisms of hyperbolic groups. I. The Hopf property, Topology 38 (1999), no. 2, 301-321.

[Szc98] A. Szczepański, Relatively hyperbolic groups, Michigan Math. J. 45 (1998), no. 3, 611-618.

[Tol93] D. Toledo, Projective varieties with non-residually finite fundamental group, Inst. Hautes Études Sci. Publ. Math. (1993), no. 77, 103-119.

[Tuk94] P. Tukia, Convergence groups and Gromov's metric hyperbolic spaces, New Zealand J. Math. 23 (1994), no. 2, 157-187.

[Wit] D. Witte, Introduction to arithmetic groups, arXiv:math/0106063v2.

[Yam04] A. Yaman, A topological characterisation of relatively hyperbolic groups, J. Reine Angew. Math. 566 (2004), 41-89.

Igor Belegradek

School of Mathematics 
Georgia Institute of Technology

Atlanta, GA 30332-0160

E-mail: ib@math.gatech.edu 